\title{
Isonicotinamide extends yeast chronological lifespan through a mechanism that diminishes nucleotides
}

Agata Kalita ${ }^{1}$, Elisa Enriquez Hesles ${ }^{1}$, Lindsey N. Power ${ }^{1}$, Dezhen Wang ${ }^{2}$, Pankaj K. Singh ${ }^{2}$, and Jeffrey S. Smith ${ }^{1 *}$

${ }^{1}$ Department of Biochemistry and Molecular Genetics, University of Virginia School of Medicine, Charlottesville, VA 22908; ${ }^{2}$ Eppley Institute for Research in Cancer and Allied Diseases, University of Nebraska Medical Center, Omaha, NE 68198

*Corresponding Author

Jeffrey S. Smith

Department of Biochemistry and Molecular Genetics

University of Virginia School of Medicine

1340 Jefferson Park Ave.

Pinn Hall, Room 6229

Charlottesville, VA 22908

Phone: 434-243-5864

Email: jss5y@virginia.edu 


\begin{abstract}
Chronological lifespan (CLS) of budding yeast, Saccharomyces cerevisiae, is a commonly utilized model for cellular aging of non-dividing cells such as neurons. CLS is strongly extended by isonicotinamide (INAM), a non-metabolized isomer of the $\mathrm{NAD}^{+}$precursor nicotinamide (NAM), but the underlying mechanisms of lifespan extension remain uncharacterized. To identify potential biochemical INAM targets, we performed a chemical genetic screen with the yeast gene knockout (YKO) strain collection for INAM-hypersensitive mutants. Significantly enriched Gene Ontology terms that emerged included SWR1 and other transcription elongation factors, as well as metabolic pathways converging on one-carbon metabolism and contributing to nucleotide biosynthesis, together suggesting that INAM perturbs nucleotide pools. In line with this model, INAM effects on cell growth were synergistic with mycophenolic acid (MPA), which extends lifespan by reducing guanine nucleotide pools. Direct measurements of nucleotides and precursors by mass spectrometry indicated that INAM reduced nucleotides, including cAMP, at 24- and 96-hour time points post-inoculation. Taken together, we conclude that INAM extends CLS by perturbing nucleotide metabolism, which may be a common functional feature of multiple anti-aging interventions.
\end{abstract}

\title{
Keywords:
}

chronological aging/isonicotinamide/nucleotides/one-carbon metabolism/yeast 


\section{INTRODUCTION}

Aging is a multifaceted process leading to decreased functioning over time, and ultimately, death of the cell or organism. Aging is also a major risk factor for many incapacitating conditions such as cardiovascular disease, cancer, and neurodegeneration (Niccoli \& Partridge, 2012). Despite extraordinary variance in organism lifespans, many of the proteins and pathways involved in aging are evolutionary conserved (Smith et al, 2008; Bitto et al, 2015). This creates the possibility of identifying lifespan-extending mechanisms and interventions in model organisms that could be effective in understanding and combating human aging and associated disease.

Environmental interventions are promising in terms of potential application in human aging. Some of them, however, such as caloric restriction (CR), are difficult to implement in humans and can reduce the subjective quality of life (Dirks \& Leeuwenburgh, 2006). Highly desired are interventions that do not require major changes in lifestyle, such as beneficial nutraceuticals or pharmaceuticals. Ideally, these types of molecules should be small, easily absorbed, and have limited negative side effects.

The budding yeast system has been effective in the identification and characterization of several such small molecule effectors of longevity, including the TOR inhibitor rapamycin (Powers et al, 2006). This unicellular eukaryote is highly suitable for testing environmental interventions using high throughput screening approaches due to its unicellular organization, short lifespan, and genetic malleability (Gershon \& Gershon, 2000). Two types of aging can be studied in S. cerevisiae: chronological (Longo et al, 2012) and replicative (Mortimer \& Johnston, 1959). Their corresponding longevity metrics are defined, respectively, as the survival of non-proliferating cells in stationary phase (CLS, chronological life span) (Longo et al, 2012) and the number of divisions that mother cells undergo before senescing (RLS, replicative life 
span) (Mortimer \& Johnston, 1959). Out of the two paradigms, chronological aging is more akin to the aging of post-mitotic cells in mammalian organisms (such as neurons) (MacLean et al, 2001), whereas replicative aging is a better model for the aging of rapidly dividing, mitotically active cells (e.g. skin stem cells) (Janssens \& Veenhoff, 2016). CLS is more accurately described as the survival of non-proliferating yeast cells in stationary phase, where they eventually lose the ability to re-enter the cell cycle, even when provided with fresh nutrients (Postnikoff \& Harkness, 2014). The CLS system is therefore also used as a model for quiescence, since it directly tests regrowth capacity of non-dividing cells (Mohammad et al, 2020). Importantly, CLS is highly dependent on environmental factors, such as media composition and carbon source levels, as well as the genetic strain background (Santos et al, 2020; Smith et al, 2016). Herein, we take advantage of these features of CLS to study the mechanism of another longevity promoting molecule, isonicotinamide (INAM).

Nicotinamide (NAM) is a water-soluble form of vitamin B3, a byproduct of the reaction catalysed by $\mathrm{NAD}^{+}$-dependent protein deacetylases (Sirtuins), and inhibits the deacetylation reaction for these enzymes through a feedback mechanism (Sauve \& Schramm, 2003; Bitterman et al, 2002). INAM is an isomer of NAM commonly used for material synthesis and pharmaceutical development due to its excellent cocrystallization properties that improve drug bioavailability (Sánchez-Férez et al, 2019; Báthori et al, 2011). INAM does not occur in cells naturally, but due to its structural similarity to NAM, has the potential for targeting NAMproducing enzymes. Indeed, NAM and INAM both act as PARP-1 inhibitors in mammalian cells (Sims et al, 1982). Of the two molecules, NAM function has been better characterized in yeast and other systems, with relatively little known about INAM. 
INAM, although structurally very similar to NAM, appears to elicit opposite effects to NAM in aging studies. In S. cerevisiae, NAM inhibition of Sir2 causes transcriptional silencing defects and destabilizes the ribosomal DNA (rDNA) locus, which drastically reduces RLS (Bitterman et al, 2002). INAM, however, functions as a Sir2 activating compound in vitro by relieving NAM inhibition (Sauve et al, 2005). INAM also promotes Sir2 function and RLS in vivo by indirectly maintaining elevated intracellular $\mathrm{NAD}^{+}$levels under conditions where $\mathrm{NAD}^{+}$ is normally low, such as media lacking nicotinic acid (McClure et al, 2012). While INAM extension of RLS requires Sir2, INAM extends CLS in a sirtuin-independent manner (Wierman et al, 2015). Furthermore, unlike CLS extension induced by restriction of glucose or methionine, the INAM-induced longevity phenotype is not dependent on Snf1 (AMPK) activity (Wierman et al, 2017). The biochemical mechanisms of CLS extension by INAM treatment therefore remain uncertain, though INAM-treated cells do have increased resistance to acetic acid compared to controls (Wierman et al, 2015), which can be beneficial for CLS (Burtner et al, 2009). Consistent with this finding, transcriptome analysis previously revealed that INAM upregulates genes involved in cell wall synthesis (Wierman et al, 2015). However, because this RNA expression data was obtained very late in stationary phase (day 8), these changes in the transcriptional landscape might not reflect the primary mechanism and target of INAM, but rather the long-term consequences of its actions.

To better understand the primary mechanisms of INAM-induced lifespan extension, in this study we utilized an unbiased chemical genomics screening approach with the yeast knockout (YKO) strain collection to identify gene deletions that confer hypersensitivity to sublethal concentrations of INAM that normally extend CLS. The idea was that such genes may point toward biochemical pathways or cellular processes that INAM perturbs. The screen 
unexpectedly implicated genes involved in transcriptional elongation and metabolic pathways related to one-carbon metabolism and nucleotide synthesis, leading to the conclusion that INAMmediated extension of CLS involves reduction of nucleotide pools.

\section{MATERIALS and METHODS}

\section{Yeast strains and media}

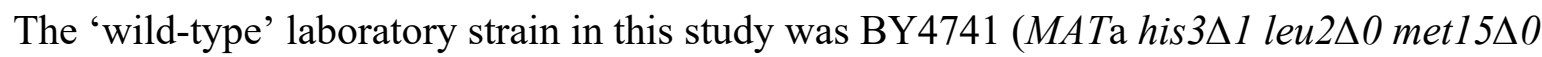

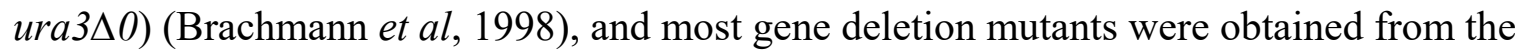
isogenic yeast knockout (YKO) collection (Winzeler et al, 1999). Detailed strain information is provided in Supplemental Table S1. Synthetic complete (SC) media with $2 \%$ glucose as a carbon source was used for most assays, with YPD used for cell propagation and selection prior to the experiments. For deletion strains with a KanMX cassette replacing the gene of interest, G418 was added to YPD at a final concentration of $200 \mathrm{mg} / \mathrm{L}$. INAM and mycophenolic acid (MPA) were supplemented into SC media at the indicated concentrations. All liquid cultures and agar plates were grown at $30^{\circ} \mathrm{C}$.

\section{CLS assays}

$10 \mathrm{ml}$ of SC media with $2 \%$ glucose and relevant supplements was inoculated with $100 \mu 1$ of overnight culture. Liquid cultures were incubated on a rotating drum (TC-7, New Brunswick Scientific) in glass tubes with metal caps allowing for gas exchange. After 72 hours, the first measurement of colony forming units on YPD agar plates was made and this was treated as day 3 for the experiment (100\% starting viability), to which all the other data was normalized.

Measurements were taken every 3-4 days as previously described (Enriquez-Hesles et al, 2021; Wierman et al, 2017). At each time point, $20 \mu \mathrm{l}$ of the cell suspension was removed from each tube and 10 -fold serially diluted three times with sterile water. Next, $2.5 \mu 1$ of each dilution 
$(1: 10,1: 100,1: 1000)$ was spotted onto a YPD plate. After 16 hours, images of the spots were taken under a Nikon Eclipse E400 brightfield microscope at 30x magnification. Microcolonies within the spots were automatically counted from the digital images using OrganoSeg (Borten et $a l, 2018)$, with the parameters adjusted for yeast colony counting (Enriquez-Hesles et al, 2021). After accounting for the dilution factor, colony numbers from each day were divided by the number of colonies from the first time point (day 3) to give the viability metrics.

\section{SGA screen}

The MATa haploid YKO collection, consisting of 4648 strains organized into 18 plates in 384well format, was spotted onto Nunc Omnitray single-well plates containing YPD-agar with 200 $\mu \mathrm{g} / \mathrm{ml} \mathrm{G} 418$ using a floating pin manual replicator (VP 384FS, V\&P Scientific). After 3 days growth, the replicator was used to transfer cells from colonies on the YPD plates to SC-agar Omnitrays supplemented with $0,25,50,75$, or 125 mM INAM. Colony growth for the $0,25,50$, and 75 mM INAM plates was imaged with a Fluor Chem Q gel documentation system (ProteinSimple) after 2 days, while the $125 \mathrm{mM}$ plates were imaged after 3 days incubation. The screen was performed in duplicate. SGAtools (http://sgatools.ccbr.utoronto.ca/) was then used to quantify colony growth from jpeg images, comparing the INAM-containing plates to control plates without INAM (Wagih et al, 2013). The score value of -0.3 was used as a cutoff for INAM sensitivity, and to limit false positives we required that both replicates reach this threshold for at least the 75- and 125-mM conditions. Most mutants with deletions of dubious open reading frames and genes without a confirmed function were omitted in further analysis, as their sensitivity would not provide any valuable information about the mechanism of action of INAM. Mutants with a score greater than +0.8 in both experiments at $125 \mathrm{mM}$ INAM were considered for the identification of resistant strains. 


\section{Growth assays}

For qualitative spots test growth assays, strains were patched onto YPD agar plates and grown overnight. The cell patches were resuspended in water at an $\mathrm{OD}_{600}$ of 1.0 , then 10 -fold serially diluted. Next, $2.5 \mu 1$ of each dilution was spotted onto SC agar plates containing $0,25,50,75$, or $125 \mathrm{mM}$ INAM, and photos taken after 2 days of growth.

For $\mathrm{IC}_{50}$ assays, $10 \mathrm{ml} \mathrm{SC}$ cultures were incubated overnight in the roller drum, then added to wells of a 96-well plate at a starting $\mathrm{OD}_{600}$ of 0.1 in $\mathrm{SC}$ media containing a range of INAM concentrations (0-240 mM; 2-fold dilutions) and other supplements as indicated. The initial $\mathrm{OD}_{600}$ was measured with a Spectra Max M2 plate reader (Molecular Devices). Plates were then sealed with a sterile, gas-permeable membrane and incubated in a heated microplate shaker (Southwest Science, SBT1500-H) at $30^{\circ} \mathrm{C}$ for $8 \mathrm{hr}$ at $900 \mathrm{RPM}$. Final $\mathrm{OD}_{600}$ was measured and the initial $\mathrm{OD}_{600}$ subtracted. Values were then normalized to fit the range $0-100 \%$, where the control condition (no INAM) was treated as $100 \%$ and the highest INAM concentration as 0 . These normalized growth values were then plotted on a graph using Origin 2018 (OriginLab, Northampton, MA, USA) and the sigmoidal fit was used to model a doseresponse curve fitting the obtained data. $\mathrm{IC}_{50}$ was calculated as the concentration at which there was $50 \%$ viability. The $95 \%$ confidence intervals were also calculated by the Origin software from the $95 \%$ confidence bands. An additional benefit of this $\mathrm{IC}_{50}$ approach is precisely determining if mutant strains with intrinsically slow growth are sensitive to INAM, due to the assay being internally normalized to growth of a given strain in the control condition (no INAM), thus allowing direct comparison of strains with different growth rates.

Synergy assessment was performed similarly to the $\mathrm{IC}_{50}$ assays, with a combination of increasing MPA and INAM concentrations added in a matrix to a 96-well plate. Methanol 
vehicle concentration was adjusted to be the same at all MPA concentrations used.

SynergyFinder (Ianevski et al, 2020) was used to analyze the synergy between compounds using baseline correction and a zero interaction potency (ZIP) model of synergy determination (Yadav et al, 2015).

\section{Measurement of metabolite abundance by mass spectrometry}

Cells were grown in $10 \mathrm{ml} \mathrm{SC} 2 \%$ glucose with or without $25 \mathrm{mM}$ INAM on a roller drum. Cultures were started from overnight liquid cultures at $\mathrm{OD}_{600}$ adjusted to 0.05 . Cell densities of cultures were measured before collection for normalization of metabolites. Cells were collected at log phase ( $\sim 5 \mathrm{hr}), 24 \mathrm{hr}$, and $96 \mathrm{hr}$. Intracellular metabolites were isolated as previously described (Shukla et al, 2015), with the extraction protocol modified for yeast. From these cultures, $9 \mathrm{ml}$ was added to $20 \mathrm{ml}$ of $100 \%$ cold $\left(-80^{\circ} \mathrm{C}\right) \mathrm{LC} / \mathrm{MS}$-grade methanol kept on dry ice (final methanol concentration: $\sim 70 \% \mathrm{v} / \mathrm{v}$ ) (Canelas et al, 2008). Samples were then kept on dry ice unless indicated otherwise. The quenched cell suspensions were spun down at $2000 \times \mathrm{g}$ in an Eppendorf $5810 \mathrm{R}$ centrifuge cooled to $-9^{\circ} \mathrm{C}$ for $5 \mathrm{~min}$. The cell pellets were resuspended in $1 \mathrm{ml}$ $80 \%$ methanol (LC/MS grade) and transferred to screw-top microfuge tubes containing $0.5 \mathrm{~mm}$ acid-washed glass beads. A Mini-Beadbeater (Biospec products) was used to vigorously shake the microfuge tubes at $4^{\circ} \mathrm{C}$ for 3 cycles of $45 \mathrm{sec}$ with 30 -second pauses. Each tube was then punctured at the bottom with a needle, placed into a glass tube and spun down at $-9^{\circ} \mathrm{C}$ for 5 minutes at 2000 RPM in the Eppendorf 5810R centrifuge to collect cell extracts. Beads were washed twice with $1.5 \mathrm{ml} \mathrm{80 \%} \mathrm{methanol} \mathrm{and} \mathrm{spun} \mathrm{down} \mathrm{the} \mathrm{same} \mathrm{way} \mathrm{(4} \mathrm{ml} \mathrm{total} \mathrm{pooled}$ volume). The pellet was resuspended by vortexing and kept on ice for 15 minutes, followed by centrifugation at $3100 \times \mathrm{g}$. Supernatants were transferred to two 2-ml microfuge tubes, and 
evaporated in a Vacufuge ${ }^{\mathrm{TM}}$ Concentrator 5301 (Eppendorf, Hamburg, Germany) at $30^{\circ} \mathrm{C}$ until $20 \%$ volume remained. Remaining water was then removed by lyophilization.

\section{Mass spectrometry}

Metabolites were analyzed as described previously (Moshfegh et al, 2019; Gunda et al, 2016) with a selected reaction monitoring (SRM) LC-MS/MS method with positive/negative ion polarity switching using a Xevo TQ-S mass spectrometer. MassLynx 4.1 software was used to calculate the peak areas for each metabolite. Peak areas were normalized to respective $\mathrm{OD}_{600}$ of each sample. These normalized peaks were subsequently scaled relative to the mean peak area in the control condition at each time point.

\section{RNA isolation and quantitative RT-PCR}

Total RNA was isolated from yeast cells harvested at log phase, $24 \mathrm{hr}$, or $96 \mathrm{hr}$ using the acidic phenol method (Collart \& Oliviero, 2001). cDNA was then synthesized from $1 \mu \mathrm{g}$ total RNA using a Verso cDNA Synthesis Kit (Thermo Scientific), and quantitated by real-time PCR reactions using SensiMix SYBR Hi-ROX Mastermix (Bioline) run on a StepOne Plus thermocycler (Applied Biosystems). Transcript levels of tested genes were normalized to levels of TAF10 mRNA (Wierman et al, 2015). Sequences of primers used for qPCR are provided in Table S2.

\section{RESULTS}

\section{INAM-induced CLS extension is titratable and independent of auxotrophies}

INAM is highly similar in chemical structure to NAM (Figure 1A), but not directly utilized as an $\mathrm{NAD}^{+}$precursor (McClure et al, 2012). To begin characterizing the mechanism of 
INAM-induced CLS extension, we first tested for dosage effects on the designer deletion strain BY4741, which contains several auxotrophies. Previous in vivo experiments with INAM were performed at a concentration of $25 \mathrm{mM}$ due to its beneficial effect on Sir2-dependent transcriptional silencing (Sauve et al, 2005). In Figure 1B, INAM was titrated into CLS cultures at concentrations ranging from 2.5 to $50 \mathrm{mM}$, inducing moderate lifespan extension at $2.5 \mathrm{mM}$ and maximal extension at $25 \mathrm{mM}$. INAM at $50 \mathrm{mM}$ did not provide added benefit and actually caused mild toxicity at early time points. Since auxotrophic mutations in BY4741 significantly impacted CLS in previous studies (Gomes et al, 2007; Smith et al, 2016), we also confirmed that INAM at the optimal $25 \mathrm{mM}$ concentration extended CLS of the related prototrophic strain, FY4 (Figure 1C). Furthermore, 25 mM INAM extended CLS of BY4741 when added to cultures at day 4 , after stationary phase entry (Figure 1D). The effect was not as strong as adding INAM at the time of inoculation, but still suggested the underlying mechanism impacts both proliferating and quiescent cells.

\section{INAM extends CLS independently of SIR2 and HST1}

Our group previously reported that Sir2 was not required for CLS extension by INAM (Wierman et al, 2015), which was unexpected given the positive effect of INAM on NAD ${ }^{+}$levels as cells enter the diauxic shift (McClure et al, 2012). Deleting SIR2 slightly extended CLS in SC media (Fabrizio et al, 2005; Smith et al, 2007), while deleting its paralog, HST1, slightly shortened it (Smith et al, 2007). Given that significant functional redundancy exists between Sir2 and Hst1

(Fine et al, 2019; Hickman \& Rusche, 2007; Li et al, 2013; Lamming et al, 2005), we hypothesized that deleting both sirtuins may block the INAM effect on CLS. As shown in Figure $1 \mathrm{E}, \mathrm{CLS}$ of a sir2 $\Delta$ ht $1 \Delta$ double mutant without INAM addition was strongly reduced compared 
to WT (BY4741), indicating that SIR2 and HST1 are indeed redundant in promoting CLS.

However, $25 \mathrm{mM}$ INAM still significantly improved lifespan of the $\operatorname{sir} 2 \Delta$ ht $1 \Delta$ strain by a similar percentage as observed for the WT strain (Figure 1E; statistics for all CLS assays are provided in Table S3), confirming that INAM works independently of Sir2 and Hst1 in the CLS system, while leaving the actual mechanism of INAM extension still unknown.

\section{Chemical genomic screen reveals INAM sensitive mutant strains}

In order to identify pathways affected by INAM, and thus provide insights about mechanisms of CLS extension, we took a non-targeted synthetic lethality screening approach using the yeast knockout (YKO) strain collection. We noticed that $50 \mathrm{mM}$ INAM reduced the initial viability of BY4741 in CLS assays (Figure 1B), consistent with slightly reduced colony size in spot tests (Figure 2A). Higher INAM concentrations slowed colony growth even further (Figure 2A), suggesting the compound was perturbing one or more pathways important for cellular growth. We therefore hypothesized that applying INAM to the KO collection strains would identify clusters of hypersensitive mutants lacking genes that buffer INAM-impaired essential pathways or processes. Such an approach was successfully utilized in a number of screens for deletion mutants hypersensitive to specific chemicals, including the CLS-extending compound rapamycin (Xie et al, 2005). An analogous screen was also performed with nicotinamide (NAM) to identify sirtuin-dependent processes, implicating pathways that prevent genomic instability (Choy et al, 2016).

The MATa haploid YKO strain collection was arrayed as colonies onto Nunc Omnitray plates containing YPD agar, then replicated onto SC 2\% glucose agar plates containing 0, 25, 50, 75, or 125 mM INAM using a 384-pin applicator tool (Figure 2B). After 2 days, the growth of 
each mutant on INAM-containing plates was quantitated relative to SC control plates using SGAtools (Wagih et al, 2013). An example of growth inhibition is shown for tho2 $\Delta$ in Figure 2C (center colony). Note that growth of all strains was strongly reduced at $125 \mathrm{mM}$, similar to spot assay results in Figure 2A. The full screen was independently performed twice, showing significant correlation in SGAtools-generated scores, as shown for $75 \mathrm{mM}$ INAM in Figure 2D $(\mathrm{R}=0.42)$. We focused on mutants with sensitivity scores lower than -0.3 in both screen replicates (Tables S4 and S5), as shown for the 240 such mutants from the $75 \mathrm{mM}$ dataset in Figure 2D (inset), fewer mutants met this stringent score criteria at $50 \mathrm{mM}(173)$, and $25 \mathrm{mM}$ (150), which was expected as the degree of pathway disruption by INAM should be weaker at lower concentrations. Only 39 genes were identified from all four INAM concentrations, though there were greater overlaps for any two concentrations (Figure 2E). The 125 mM INAM concentration caused a strong growth defect with the BY4741 control and most mutants (Figures $2 \mathrm{~A}$ and $\mathrm{C}$ ), and $\sim 50 \%$ of the 326 mutants sensitive to this concentration did not overlap with mutants from the other INAM concentrations (Figure 2E), suggesting there could be numerous false positive hits in this portion of the screen. We therefore focused Gene Ontology (GO) enrichment analysis on a collection of 341 genes identified from at least one of the 25,50 , or 75 mM INAM conditions (Table S4). Several 'Biological Process' GO terms with significant pvalues were related to transcriptional regulation, vacuolar transport, inositol phosphate biosynthesis, and chromatin remodelling (Table S6). Significant 'Cellular Component' GO terms covered the INO80 and SWR1 chromatin remodelling complexes, transcriptional elongation factors, endosomes and the ESCRT complex (Table S6). With this functional information in hand, we chose 61 candidate mutants for verification of INAM sensitivity using individual spot test growth assays at 25, 50, and $75 \mathrm{mM}$ (Figures $\mathrm{S} 1$ and S2). The mutants selected at this point 
all had SGAtools scores lower than -0.3. INAM sensitivity was confirmed for 57 of 61 re-tested deletion mutants (Figure S1 and S2), yielding a high positive predictive value (PPV) of 93.4\%. This high PPV also indicated the sensitivity cutoff score of -0.3 was highly stringent, suggesting we likely missed some false-negative mutants. Therefore, based on the enriched GO terms in Table S6 and the literature, we were able to identify 22 additional INAM-sensitive deletion mutants (Figure S3).

\section{Cells lacking BNA7 are resistant to high INAM concentrations}

Colony growth was strongly reduced by $125 \mathrm{mM}$ INAM (Figure 2A and C), potentially allowing for identification of INAM-resistant-mutants in the screen. While there were no significantly enriched GO-terms identified among the mutants picked as resistant to $125 \mathrm{mM}$ INAM (Table S5; 0.8 cutoff score), we still tested 20 of these candidates for INAM-resistance in spot test growth assays. The only mutant confirmed as resistant was bna7D (Figure S4A). We verified this result by remaking the bna $7 \Delta:: k a n M X 4$ mutant from BY4741 and then retesting the INAM-resistant phenotype. BNA7 encodes formylkynurenine formamidase, an enzyme that carries out the second step of de novo $\mathrm{NAD}^{+}$biosynthesis from tryptophan, releasing kynurenine and formate as products (Figure S4B). This was intriguing because we previously isolated bna2A in a screen for chronologically long-lived mutants (Matecic et al, 2010). However, deleting $B N A 2$, or other $B N A$ genes in this pathway other than $B N A 7$, had no effect on INAM sensitivity, indicating a unique feature of lacking the Bna7 enzyme. The bna7 4 mutant was also long-lived compared to BY4741, and 25 mM INAM significantly extended CLS even further (Figure S4C), suggesting that INAM does not primarily target the Bna7 enzyme. Therefore, the mechanism of how loss of Bna7 confers INAM resistance remains unclear, but could involve alterations in 
enzyme substrate or product levels. Interestingly, this mutant was also resistant to the phenolic compound, ferulic acid (Fletcher et al, 2019). Noteworthy, the Bna7 product, formate, is an input to the one-carbon metabolism pathway.

\section{SWR1 complex subunit mutants are sensitive to INAM}

One of the strongest enriched GO terms for INAM hypersensitive mutants was the SWR1 complex (Table S6), a conserved ATP-dependent chromatin remodeler that exchanges H2A core histones in nucleosome octamers with the H2A.Z variant encoded by HTZ1 (Figure 3A; (Santisteban et al, 2000; Mizuguchi et al, 2004)). Five SWR1 complex mutants (bdf1 1 , vps 72A, swc54, swr14, yaf94) were identified from the screen at 25, 50, or $75 \mathrm{mM}$ INAM concentrations, and all were confirmed by spot test growth assays (Figures S1-S3). We also tested three additional annotated subunit mutants found in the haploid YKO collection $(\operatorname{arp} 6 \Delta, s w c 3 \Delta$, $s w c 7 \Delta$ ), but only arp6 $\Delta$ and $s w c 3 \Delta$ were confirmed as INAM sensitive. This actually made sense because despite being part of the SWR1 complex, Swc7 was not required for H2A.Z binding or histone replacement, and had minimal contributions to complex stability (Wu et al, 2009). Importantly, the $h t z 1 \Delta$ mutant strain itself was isolated from the screen at the 50, 75, and 125 mM INAM concentrations, and also confirmed as sensitive at $25 \mathrm{mM}$ (Figure 3B). We note that $h t z 1 \Delta$ and SWR1 complex mutants were identified as sensitive to ferulic acid (Fletcher et al, 2019), highlighting another similarity between INAM and this phenolic compound.

Defects in the SWR1 complex were previously shown to extend CLS of a prototrophic strain in nitrogen (glutamine)-rich media conditions, but not nitrogen (GABA)-poor media (Garay et al, 2014). We were therefore curious if $s w r l \Delta$ or $h t z 1 \Delta$ mutants were also long-lived in SC media with ammonium sulfate nitrogen conditions used for the INAM sensitivity screen. As shown in Figure 3C, there were no significant differences in CLS between the mutants and WT 
BY4741 without INAM, and their lifespans were fully extended by $25 \mathrm{mM}$ INAM. Despite the requirement of SWR1 activity for resistance to high INAM concentrations, it was not required for CLS extension at the optimal $25 \mathrm{mM}$ concentration. We concluded that deletion mutants isolated from the INAM-sensitivity screen will not necessarily be in pathways that directly regulate CLS, or its responsiveness to INAM.

\section{Disruption of serine, cysteine and threonine metabolism confers sensitivity to INAM} Intriguing metabolism-related GO terms enriched in the 25,50 , or $75 \mathrm{mM}$ INAM-sensitive mutant lists were de novo IMP biosynthesis ( $A D E 1, A D E 4, A D E 6, A D E 5,7)$, threonine metabolic process (HOM2, HOM3, THR4, and GLY1), and serine family amino acid biosynthetic process (CYS4, GLY1, HOM2, HOM3, SER1, SER2) (Table S6). CYS3 was also identified from the 125 mM INAM concentration (Table S5). Figure 4A depicts connections between these enzymes, with the points of convergence at serine and glycine. Both of these amino acids are donors of one-carbon units in the folate/one-carbon metabolism pathway, whereby one-carbon moieties linked with tetrahydrofolate are major sources of building blocks for de novo purine synthesis (Appling, 1991). We previously reported that deletion of one-carbon metabolism and the $A D E$ genes extended CLS in SC media (Matecic et al, 2010). Moreover, serine metabolism was strongly linked to CLS regulation, as serine supplementation extended CLS (Enriquez-Hesles et al, 2021a; Maruyama et al, 2016), and inactivation of SERl was identified as a major QTL for genetic CLS variation among strain backgrounds (Jung et al, 2018). We were able to recapitulate moderate CLS extension caused by impaired serine biosynthesis with a ser $2 \Delta$ mutant (Figure 4B), but similar to the $s w r 1 \Delta$ mutant, INAM fully extended CLS of the ser $2 \Delta$ mutant (Figure 
4B). Intriguingly, one-carbon moieties from the mitochondria are transported to the cytoplasm as formate, which is also a product of the Bna7 enzymatic reaction (Figure S4B).

The $\operatorname{ser} 2 \Delta$ mutant visually showed strong sensitivity to 50 and $75 \mathrm{mM}$ INAM on SC plates, but not $25 \mathrm{mM}$ (Figure 4C). To more precisely quantify differences in sensitivity between strains, we switched from qualitative spot test growth assays to quantitative measurements of $\mathrm{IC}_{50}$ values in small liquid cultures (Figure 4D, see materials and methods for details). For BY4741, the $\mathrm{IC}_{50}$ with INAM in liquid cultures was between 60 and $70 \mathrm{mM}$ depending on the experiment (Figure $4 \mathrm{E})$. The $\mathrm{IC}_{50}$ of INAM for a ser $2 \triangle$ mutant was only $38.5 \mathrm{mM}(95 \%$ confidence intervals: $36.6-40.6 \mathrm{mM}$ ) (Figure 4E), consistent with the spot assay sensitivity results.

Having established a working $\mathrm{IC}_{50}$ assay, we employed it to test if the sensitivity of amino acid synthesis mutants such as $\operatorname{ser} 2 \Delta$ and $\operatorname{thr} 1 \Delta$ could be rescued by providing the respective amino acids. To this end, we tested INAM $\mathrm{IC}_{50}$ for BY4741 and deletion mutants when supplemented with $25 \mathrm{mM}$ serine or threonine (Figure S5). As predicted, serine rescued low $\mathrm{IC}_{50}$ of the $\operatorname{ser} 2 \Delta$ mutant without affecting BY4741. Similarly, threonine rescued low $\mathrm{IC}_{50}$ of thr $1 \Delta$ and hom $3 \Delta$ mutants defective in threonine synthesis, without affecting BY4741. These results suggested that abundant serine and threonine help cells tolerate the negative impact of high INAM concentrations.

\section{INAM acts synergistically with MPA}

Enriched GO terms for INAM sensitivity pointed to the SWR1 complex, transcriptional elongation complexes, and amino acid metabolic pathways clustering around one-carbon metabolism. Each of these terms is directly related to the availability of nucleotide pools. For 
example, cells partially impaired for transcriptional elongation, including $s w r l \Delta$ and $h t z 1 \Delta$ mutants (Santisteban et al, 2011), are sensitive to mycophenolic acid (MPA) or 6-azauracil (6AU) (Desmoucelles et al, 2002), inhibitors of inosine 5'-monophosphate dehydrogenase (IMPDH) that deplete guanine nucleotide pools (Escobar-Henriques et al, 2001; Sweeney et al, 1972; Qiu et al, 2000). We therefore compared INAM-sensitive mutants identified in our screen to previously identified MPA-sensitive strains (Desmoucelles et al, 2002). As shown in Figure 5A, there was significant overlap between the datasets, with 45.1\% (46/102) of MPA-sensitive mutants also identified as sensitive to at least one INAM concentration in the primary screen, but only $9.1 \%(46 / 504)$ of all candidate INAM-sensitive mutants, including at $125 \mathrm{mM}$, were also sensitive to MPA. To determine if such overlap might indicate a functional similarity between the compounds, perhaps even inhibiting the same target, we tested for synergy between MPA and INAM by measuring effects of combining the compounds at increasing concentrations on cellular growth in the $\mathrm{IC}_{50}$ assay. Visual representation of the synergistic parameter $\delta$ for each of the concentration combinations revealed clear synergistic effects at relatively low concentrations where they did not inhibit growth individually (Figure 5B). The strong synergistic interaction indicated that INAM and MPA likely target related pathways within an interaction network (Yin et al, 2014), but not the same enzyme, as this would lead to an additive effect.

MPA was known to extend RLS of S. cerevisiae (Liu et al, 2020; Sarnoski et al, 2017), and CLS of another yeast species, Schizosaccharomyces pombe (Stephan et al, 2013). As shown in Figure 5C, MPA also extended CLS of S. cerevisiae at $1 \mu \mathrm{M}$, and even more effectively at 10 $\mu \mathrm{M}$. This effect was completely abolished by $0.1 \mathrm{mM}$ guanine supplementation. Guanine is imported and then salvaged into GMP by hypoxanthine guanine phosphoribosyltransferase (Hpt1), thus bypassing the need for IMPDH, the target of MPA inhibition. This result confirmed 
that MPA extended CLS through depletion of guanylic nucleotides. In contrast, CLS extension by $25 \mathrm{mM}$ INAM was not prevented by guanine supplementation (Figure 5D), indicating that IMPDH was likely not a primary target of INAM, and thus consistent with the synergistic effects of MPA and INAM on cell growth.

\section{INAM modifies nucleotide metabolism}

Since CLS extension by INAM was not prevented by guanine addition, and given the involvement of serine and threonine, both of which support nucleotide pools (Kastanos et al, 1997; Hartman, 2007), we decided to test the impact of 25 mM INAM on a wider range of nucleotides, nucleosides, and nucleobases. Levels of such metabolites in extracts from cells at $\log$ phase, late diauxic shift (24 hr), or stationary phase (96 hr) were quantified by mass spectrometry. We reasoned that nucleotide changes occurring at log phase and/or $24 \mathrm{hr}$ of liquid growth would reflect changes occurring in colonies growing on agar plates, specifically inner cells of the colony are exposed to nutrient stress (Váchová et al, 2012). As shown in Figure 6A, INAM significantly reduced several bases and nucleosides during log phase, which then extended to nucleotides, including cAMP and cGMP, at 24 and 96 hrs (Figure 6A and B). By 96 hrs, some nucleotides (not the triphosphates) were elevated in INAM-treated cells compared to the control (Figure 6A). This effect was most prominent with uracil, uridine, UMP, and UDP at $96 \mathrm{hrs}$ (Figure S7A). We hypothesized this result could be related to the ura3s mutation in BY4741, which blocks UMP synthesis from orotidine-5'-phosphate and makes cells auxotrophic for uracil (Figure S7A). While supplementing uracil at 4x the normal concentration in SC media extended CLS, it was less effective than 25 mM INAM (Figure 6C). Furthermore, INAM still fully extended CLS in combination with the supplemented uracil without an additive effect. The 
ura3 $\Delta 0$ mutation in BY4741 applies pressure on cells to salvage uracil from the recycling of RNA, and INAM may augment this activity, but may also function elsewhere.

To complement the nucleotide quantification, quantitative RT-PCR was used to assay expression of several genes that function in nucleotide metabolism. As shown in Figures S6 and S7A, most tested genes showed reduced expression at 24 and 96 hrs, which was expected as cells enter stationary phase. However, INAM increased expression of RNR3 and IMD2 at 96 hrs. $R N R 3$ encodes a minor large ribonucleotide reductase subunit that is nearly undetectable under normal growth conditions, but strongly induced by DNA damaging agents, including hydroxyurea (HU) (Domkin et al, 2002). IMD2 encodes a major isoform of inosine monophosphate dehydrogenase, the target of MPA. Expression of IMD2, but not the other IMD genes, is also induced by MPA (Escobar-Henriques \& Daignan-Fornier, 2001). Both of these changes were consistent with impacts of INAM on nucleotide pools, but not indicative of a single mechanism. Interestingly, almost all the genes we tested by RT-PCR were elevated at day 8 in INAM compared to the control (Figure S7B), including $A D O 1, A A H 1, I M D 4$, and $R N R 1$, each of which was identified as upregulated by INAM in an earlier microarray transcriptomics study (Wierman et al, 2015). Taken all together, we conclude that INAM extends CLS through a mechanism that perturbs nucleotide metabolism.

\section{DISCUSSION}

\section{CLS and Sir2}

INAM first came to our attention because this synthetic product prevented feedback inhibition of Sir2 by the NAM product of its enzymatic reaction (Sauve et al, 2005). Unexpectedly, it extended RLS by indirectly maintaining elevated NAD ${ }^{+}$levels, most likely by 
increasing flux through the $\mathrm{NAD}^{+}$salvage pathway (McClure et al, 2012), potentially through a hormesis effect. When we examined the impact of INAM on CLS, it was found to strongly extend lifespan independently of Sir2 (Wierman et al, 2015). The impact of Sir2 on CLS depends on media composition and genetic strain background (Wierman \& Smith, 2014), with most lab media conditions resulting in CLS extension when Sir2 was deleted or inhibited (Casatta et al, 2013; Fabrizio et al, 2005; Orlandi et al, 2017; Smith et al, 2007). Here, we found that CLS was drastically reduced in cells lacking both SIR2 and HST1, consistent with several instances of these two paralogs compensating for each other in other assay systems (Hickman \& Rusche, 2007; Li et al, 2013). This new result unifies the positive role of Sir2 in yeast cell survival, both replicatively and chronologically, as well as with sirtuins in higher eukaryotes. Given the ability of INAM to maintain $\mathrm{NAD}^{+}$levels in cells entering stationary phase (McClure et al, 2012), we were surprised that INAM still extended CLS of the sir $2 \Delta$ hst $1 \Delta$ double mutant, although this pointed to INAM impacting CLS through a sirtuin-independent mechanism, thus leading to the screen in this study.

\section{INAM effects compared to NAM}

The sensitivity selection criteria chosen in this study were very strict in order to minimize the number of false positives that can occur with YKO screens. We know that INAM sensitive mutants were missed because out of the deletion strains selected for testing based on follow-up to primary screen hits and literature searching, 22 were sensitive to INAM, even though they did not meet the original screening criteria. Such mutants turned out to be, on average, less INAMsensitive than those identified by the original criteria (score $<-0.3$ ). This result suggests that the screen likely identified the most sensitive strains to INAM, but not all. Even at this point of the 
screen, the candidates identified were sufficient to implicate nucleotide metabolism as a likely target for perturbation by INAM.

An earlier screen performed with the YKO strain collection for deletion mutants sensitive to $120 \mathrm{mM}$ nicotinamide (NAM) identified genes that function in sister chromatid cohesion and maintenance of genome stability (Choy et al, 2016), cellular processes that were not significantly enriched in our screen for INAM-sensitive mutants. However, 21 of these 59 NAM-sensitive mutants overlapped with our list of candidate INAM-sensitive mutants from Table S4, including htz1D. From the supplemental tables (Choy et al, 2016), we also extracted NAM-sensitive mutants with normalized colony growth ratios of $<0.5$ in both screen replicates (107 genes), revealing significantly enriched GO terms of DNA repair (as expected), but also de novo IMP biosynthesis ( $A D E 1, A D E 2, A D E 4, A D E 6, A D E 8)$. Several $A D E$ gene mutants were also identified from our INAM-sensitivity screen, suggesting that NAM and INAM share some level of overlap in their physiological effects on yeast cells, as might be expected for compounds with such similar chemical structures (Figure 1A). There are also significant differences, including that NAM appears to be less toxic than INAM to yeast cells at high concentrations. Additionally, relatively low INAM concentrations $(2.5$ or $10 \mathrm{mM}$ ) extend CLS, while low NAM (1 or $5 \mathrm{mM})$ shortens CLS, most likely due to sirtuin inhibition (Smith et al, 2007). It remains possible that NAM at higher concentrations could also extend CLS similarly to INAM, thus overriding the inhibitory effect on sirtuins.

\section{Interpretation and limitations of INAM sensitivity screening}

It is important to note that the sensitivity of deletion strains to a given compound does not necessarily result from direct participation of the deleted gene in the compound-impaired pathway. For example, there are multidrug-resistance conferring genes such as non-specific 
efflux membrane pumps that make cells sensitive to a wide array of toxic compounds when deleted. In S. cerevisiae, strains with deletions of genes relevant for vacuolar structure and function, follicular transport, cell structure, and lipid metabolism are also susceptible to many compounds with diverse, unconnected modes of action (Parsons et al, 2004). Only five of all strains that we confirmed as sensitive to INAM were also sensitive to the wider range of other compounds: gal11 1 , ies64, spc724, vrp1 $\Delta$ and def1 (Parsons et al, 2004). Interestingly, several deletion strains previously identified as MPA-sensitive were also sensitive to rapamycin (Desmoucelles et al, 2002; Chan et al, 2000), three of which were sensitive to INAM in our study (ser2A, hom 2A, and $\operatorname{cdc} 10 \Delta)$. Additionally, hom $3 \Delta, \operatorname{rnr} 1 \Delta, \operatorname{ser} 1 \Delta$, and aat $2 \Delta$ mutants involved in serine, aspartate/threonine synthesis or ribonucleotide reductase activity were all sensitive to rapamycin (Chan et al, 2000). Importantly, such mutants are not generally sensitive to a broader spectrum of compounds (Parsons et al, 2004), implying there could be functional overlap between these CLS extending compounds at the level of nucleotide metabolism.

\section{INAM effects on nucleotide metabolism}

Our chemical genetic screen indicated that perturbing the pathways for serine, threonine, aspartate or cysteine synthesis conferred INAM sensitivity. Each of these pathways converge on the reaction of serine conversion to glycine, in which the methylene group of serine is incorporated into the folate cycle/one-carbon metabolism pathway by serine hydroxymethyltransferases (Kastanos et al, 1997). This pathway is the main source of onecarbon moieties in the cell, which in turn, together with glycine, provides key substrates for de novo purine nucleotide biosynthesis (Kastanos et al, 1997). The one-carbon pathway reactions can occur either in the mitochondria or in the cytoplasm, depending on the availability of nutrients (Kastanos et al, 1997). De novo purine synthesis occurs in the cytosol, so single carbon 
groups formed in the mitochondria must be exported there. The trifunctional enzyme methylenetetrahydrofolate dehydrogenase (Mis1 in yeast, MTHD2 in mammals) releases the one-carbon moiety from mitochondria in the form of formic acid, where it can be re-incorporated into the cytosolic branch of the one-carbon pathway for use in purine biosynthesis (Kastanos et al, 1997). Formic acid is also a product of the reaction catalyzed by Bna7 (Wogulis et al, 2008) a protein whose absence conferred INAM resistance. Formic acid might therefore be a metabolite that connects the observations made in this study between INAM and potential negative regulation of one-carbon metabolism. This is especially intriguing given that onecarbon metabolism is rapidly emerging as an important regulatory hub for longevity pathways (Maitra et al, 2020; Annibal et al, 2021; Enriquez-Hesles et al, 2021). Further work is necessary to explain in detail the effect of INAM on formic acid levels and one-carbon metabolism. In particular, identification of the molecular target of INAM will contribute to a better understanding of the action of this compound and its potential utility.

Unlike the effects of MPA in previous studies (Hyle et al, 2003), 25 mM INAM did not have a dramatic effect on nucleotide pools in exponentially growing cells, but the reduction in trinucleotides became noticeable in cells grown for 24 or 96 hours. We hypothesize that INAM perturbs the flux of nucleotide availability further upstream of MPA, which directly targets the IMP-dehydrogenase enzyme to produce an acute impact on guanine nucleotides. The effect of INAM on nucleotides becomes more noticeable when pools are naturally being depleted as cells enter stationary phase (Osório et al, 2005). We anticipate that the negative effect of INAM on trinucleotide pools would be stronger in proliferating cells if the concentration was sufficiently increased to slow growth rates, such as 50,75 , or $125 \mathrm{mM}$. The partial effect on nucleotides at 25 $\mathrm{mM}$ and lower could be inducing a hormesis response that is beneficial for longevity, similar to 
that proposed for MPA (Liu et al, 2020). Evidence for this idea comes from earlier findings that $25 \mathrm{mM}$ INAM prevents depletion of $\mathrm{NAD}^{+}$as cells enter stationary phase, increases expression of NAD ${ }^{+}$biosynthesis and salvage pathway genes (McClure et al, 2012), and incurs cell resistance to high exogenous acetic acid levels (Wierman et al, 2015). In this current study we also found that genes involved in the salvage of other nucleotides are induced by INAM relative to the control condition, especially at day 8 of the aging assays. Additionally, we observed that pyrimidine salvage intermediates were generally reduced in log phase cells, but became elevated in cells grown for $96 \mathrm{hr}$, suggesting upregulation of pyrimidine nucleotide salvage pathways.

\section{cAMP and chronological aging}

The strongest negative effects of INAM on nucleotides were for cAMP and cGMP at the $96 \mathrm{hr}$ timepoint. Extracellular glucose is sensed by the G-protein coupled receptor (Gpr1/Gpa2) to activate cAMP synthesis by adenylyl cyclase (Cyr1). cAMP then activates protein kinase A (PKA), which regulates cell growth, metabolism, and stress resistance. For example, PKA directly represses the key transcription factor Rim15, preventing it from entering the nucleus (Broach, 2012). Glucose restriction reduces cAMP/PKA signalling, allowing Rim15 to enter the nucleus and activate genes with Post-Diauxic Shift (PDS) or STress Response Element (STRE) motifs, which mediates RLS extension (Wei et al, 2008). cAMP levels are typically high in proliferating yeast cells and then reduced as cells enter stationary phase, controlled by the balance between the activities of adenylyl cyclase and the cAMP phosphodiesterases, Pde1 and Pde2 (Park et al, 2005). Little is known about the role of cGMP in yeast cells, but it has been proposed to help fine tune cAMP levels (Cardarelli et al, 2019). The observed cAMP reduction in INAM-treated cells at $96 \mathrm{hr}$ is consistent with lifespan extension, and suggests stimulation of Pde 2 and/or reduced Cyr1 activity. Either way, the reduction in cAMP caused by INAM could 
be enhancing stationary phase entry and quiescence maintenance, thus contributing to CLS extension.

\section{INAM effects in mouse and humans}

Could INAM be used as an intervention in humans? INAM actually inhibits the activity of PARP-1 in mammalian cells, similar to the effect of NAM (Ueda et al, 1995). No protein similar to PARP-1 in sequence or structure is encoded in the yeast genome (Citarelli et al, 2010). Due to inhibition of PARP-1, INAM has been studied in the past in mice. INAM exhibited antiinflammatory and analgesic effects (Godin et al, 2011), also protected $\beta$-cells of the pancreas from streptozotocin-induced damage and apoptosis, and from diabetes caused by such damage (Fukaya et al, 2013). Such studies were not performed in PARP-1 knockout mice as a control, so it cannot be excluded that some of these positive effects are not due to PARP-1 inhibition by INAM, but occur through a path similar to the one described in this paper.

MPA is used in immunosuppressive therapy because it inhibits the proliferation of lymphocytes (Mitsui \& Suzuki, 1969). Therefore, it is possible that the observed antiinflammatory effect of INAM is due to a decrease in nucleotide levels by this compound. There are also data relevant to safety of INAM. For example, it has been shown that administration of this compound throughout the lifespan of mice does not cause increased cancer incidence (Toth, 1983). Unfortunately, this 1983 publication did not measure life expectancy data for the control group, which makes it impossible to assess the impact of INAM on lifespan in this experiment. Future INAM studies in metazoan organisms therefore appear warranted. 


\section{ACKNOWLEDGMENTS}

We thank members of the Smith lab, David Auble, and Marty Mayo for helpful discussions.

E.E.H was supported by the Medical Scientist Training Program (MSTP) training grant T32GM007267, the Cell and Molecular Biology (CMB) training grant T32GM008136, and an individual National Research Service Award from the NIH, F30AG067760. The study was supported by NIH grants RO1GM075240 and RO1GM127394 to J.S.S., as well as RO1CA216853 and RO1CA163649 to P.K.S.

\section{REFERENCES}

Annibal A, Tharyan RG, Schonewolff MF, Tam H, Latza C, Auler MMK \& Antebi A (2021)

Regulation of the one carbon folate cycle as a shared metabolic signature of longevity. Nat Commun 12: 3486

Appling DR (1991) Compartmentation of folate-mediated one-carbon metabolism in eukaryotes. FASEB J 5: 2645-2651

Báthori NB, Lemmerer A, Venter GA, Bourne SA \& Caira MR (2011) Pharmaceutical Cocrystals with isonicotinamide-vitamin B3, clofibric acid, and diclofenac-and two isonicotinamide hydrates. Cryst Growth Des 11: 75-87

Bitterman KJ, Anderson RM, Cohen HY, Latorre-Esteves M \& Sinclair DA (2002) Inhibition of silencing and accelerated aging by nicotinamide, a putative negative regulator of yeast Sir2 and human SIRT1. J Biol Chem 277: 45099-45107

Bitto A, Wang AM, Bennett CF \& Kaeberlein M (2015) Biochemical Genetic Pathways that 
Modulate Aging in Multiple Species: Figure 1. Cold Spring Harb Perspect Med 5: a025114

Borten MA, Bajikar SS, Sasaki N, Clevers H \& Janes KA (2018) Automated brightfield morphometry of 3D organoid populations by OrganoSeg. Sci Rep 8: 1-10

Brachmann CB, Davies A, Cost GJ, Caputo E, Li J, Hieter P \& Boeke JD (1998) Designer deletion strains derived from Saccharomyces cerevisiae S288C: A useful set of strains and plasmids for PCR-mediated gene disruption and other applications. Yeast 14: 115-132

Broach JR (2012) Nutritional control of growth and development in yeast. Genetics 192: 73-105

Burtner CR, Murakami CJ, Kennedy BK \& Kaeberlein M (2009) A molecular mechanism of chronological aging in yeast. Cell Cycle 8: 1256-1270

Canelas AB, Ras C, ten Pierick A, van Dam JC, Heijnen JJ \& van Gulik WM (2008) Leakagefree rapid quenching technique for yeast metabolomics. Metabolomics 4: 226-239

Cardarelli S, Giorgi M, Poiana G, Biagioni S \& Saliola M (2019) Metabolic role of cGMP in S. cerevisiae: the murine phosphodiesterase- 5 activity affects yeast cell proliferation by altering the cAMP/cGMP equilibrium. FEMS Yeast Res 19: foz016

Casatta N, Porro A, Orlandi I, Brambilla L \& Vai M (2013) Lack of Sir2 increases acetate consumption and decreases extracellular pro-aging factors. Biochim Biophys Acta - Mol Cell Res 1833: 593-601

Chan TF, Carvalho J, Riles L \& Zheng XFS (2000) A chemical genomics approach toward understanding the global functions of the target of rapamycin protein (TOR). Proc Natl Acad Sci U S A 97: 13227-13232

Choy JS, Qadri B, Henry L, Shroff K, Bifarin O \& Basrai MA (2016) A genome-wide screen 
with nicotinamide to identify sirtuin-dependent pathways in Saccharomyces cerevisiae. G3

Genes, Genomes, Genet 6: 485-494

Citarelli M, Teotia S \& Lamb RS (2010) Evolutionary history of the poly(ADP-ribose)

polymerase gene family in eukaryotes. BMC Evol Biol 10: 1-26

Collart MA \& Oliviero S (2001) Preparation of yeast RNA. Curr Protoc Mol Biol Chapter 13:

Unit13.12

Desmoucelles C, Pinson B, Saint-Marc C \& Daignan-Fornier B (2002) Screening the yeast

'Disruptome' for mutants affecting resistance to the immunosuppressive drug, mycophenolic acid. J Biol Chem 277: 27036-27044

Dirks AJ \& Leeuwenburgh C (2006) Caloric restriction in humans: Potential pitfalls and health concerns. Mech Ageing Dev 127: 1-7

Domkin V, Thelander L \& Chabes A (2002) Yeast DNA damage-inducible Rnr3 has a very low catalytic activity strongly stimulated after the formation of a cross-talking Rnr1/Rnr3 complex. J Biol Chem 277: 18574-18578

Enriquez-Hesles E, Smith Jr DL, Maqani N, Wierman MB, Sutcliffe MD, Fine RD, Kalita A, Santos SM, Muehlbauer MJ, Bain JR, et al (2021) A cell-nonautonomous mechanism of yeast chronological aging regulated by caloric restriction and one-carbon metabolism. $J$ Biol Chem 296: 100125

Escobar-Henriques M, Balguerie A, Monribot C, Boucherie H \& Daignan-Fornier B (2001) Proteome analysis and morphological studies reveal multiple effects of the immunosuppressive drug mycophenolic acid specifically resulting from guanylic nucleotide 
depletion. J Biol Chem 49: 46237-46242

Escobar-Henriques M \& Daignan-Fornier B (2001) Transcriptional regulation of the yeast GMP synthesis pathway by its end products. J Biol Chem 276: 1523-1530

Fabrizio P, Gattazzo C, Battistella L, Wei M, Cheng C, McGrew K \& Longo VD (2005) Sir2 blocks extreme life-span extension. Cell 123: 655-667

Fine RD, Maqani N, Li M, Franck E \& Smith JS (2019) Depletion of limiting rDNA structural complexes triggers chromosomal instability and replicative aging of Saccharomyces cerevisiae. Genetics 212: 75-91

Fletcher E, Gao K, Mercurio K, Ali M \& Baetz K (2019) Yeast chemogenomic screen identifies distinct metabolic pathways required to tolerate exposure to phenolic fermentation inhibitors ferulic acid, 4-hydroxybenzoic acid and coniferyl aldehyde. Metab Eng 52: 98109

Fukaya M, Tamura Y, Chiba Y, Tanioka T, Mao J, Inoue Y, Yamada M, Waeber C, IdoKitamura Y, Kitamura T, et al (2013) Protective effects of a nicotinamide derivative, isonicotinamide, against streptozotocin-induced $\beta$-cell damage and diabetes in mice. Biochem Biophys Res Commun 442: 92-98

Garay E, Campos SE, González de la Cruz J, Gaspar AP, Jinich A \& DeLuna A (2014) Highresolution profiling of stationary-phase survival reveals yeast longevity factors and their genetic interactions. PLoS Genet 10: e1004168

Gershon H \& Gershon D (2000) The budding yeast, Saccharomyces cerevisiae, as a model for aging research: A critical review. Mech Ageing Dev 120: 1-22 
Godin AM, Ferreira WC, Rocha LTS, Seniuk JGT, Paiva ALL, Merlo LA, Nascimento EB, Bastos LFS \& Coelho MM (2011) Antinociceptive and anti-inflammatory activities of nicotinamide and its isomers in different experimental models. Pharmacol Biochem Behav 99: $782-788$

Gomes P, Sampaio-Marques B, Ludovico P, Rodrigues F \& Leão C (2007) Low auxotrophycomplementing amino acid concentrations reduce yeast chronological life span. Mech Ageing Dev 128: 383-391

Gunda V, Yu F \& Singh PK (2016) Validation of metabolic alterations in microscale cell culture lysates using hydrophilic interaction liquid chromatography (HILIC)-tandem mass spectrometry-based metabolomics. PLoS One 11: e0154416

Hartman 4th JL (2007) Buffering of deoxyribonucleotide pool homeostasis by threonine metabolism. Proc Natl Acad Sci U S A104: 11700-11705

Hickman MA \& Rusche LN (2007) Substitution as a mechanism for genetic robustness: The duplicated deacetylases Hst1p and Sir2p in Saccharomyces cerevisiae. PLoS Genet 8: e126

Hyle JW, Shaw RJ \& Reines D (2003) Functional distinctions between IMP dehydrogenase genes in providing mycophenolate resistance and guanine prototrophy to yeast. $J$ Biol Chem 278: $28470-28478$

Ianevski A, Giri AK \& Aittokallio T (2020) SynergyFinder 2.0: visual analytics of multi-drug combination synergies. Nucleic Acids Res 48: W488-W493

Janssens GE \& Veenhoff LM (2016) Evidence for the hallmarks of human aging in replicatively aging yeast. Microb Cell 3: 263-274 
Jung PP, Zhang Z, Paczia N, Jaeger C, Ignac T, May P \& Linster CL (2018) Natural variation of chronological aging in the Saccharomyces cerevisiae species reveals diet-dependent mechanisms of life span control. NJP Aging Mech Dis 12: 3

Kastanos EK, Woldman YY \& Appling DR (1997) Role of mitochondrial and cytoplasmic serine hydroxymethyltransferase isozymes in de novo purine synthesis in Saccharomyces cerevisiae. Biochemistry 36: 14956-14964

Lamming DW, Latorre-Esteves M, Medvedik O, Wong SN, Tsang FA, Wang C, Lin SJ \& Sinclair DA (2005) Cell biology: HST2 mediates SIR2-independent life-span extension by calorie restriction. Science 309: 1861-1864

Li M, Valsakumar V, Poorey K, Bekiranov S \& Smith JS (2013) Genome-wide analysis of functional sirtuin chromatin targets in yeast. Genome Biol 14: R48

Liu P, Sarnoski EA, Olmez TT, Young TZ \& Murat Acar · (2020) Characterization of the impact of GMP/GDP synthesis inhibition on replicative lifespan extension in yeast. Curr Genet 1: 3

Longo VD, Shadel GS, Kaeberlein M \& Kennedy B (2012) Replicative and chronological aging in Saccharomyces cerevisiae. Cell Metab 16: 18-31

MacLean M, Harris N \& Piper PW (2001) Chronological lifespan of stationary phase yeast cells; a model for investigating the factors that might influence the ageing of postmitotic tissues in higher organisms. Yeast 18: 499-509

Maitra N, He C, Blank HM, Tsuchiya M, Schilling B, Kaeberlein M, Aramayo R, Kennedy BK \& Polymenis M (2020) Translational control of one-carbon metabolism underpins ribosomal protein phenotypes in cell division and longevity. Elife 9: e53127 
Maruyama Y, Ito T, Kodama H \& Matsuura A (2016) Availability of amino acids extends chronological lifespan by suppressing hyper-acidification of the environment in Saccharomyces cerevisiae. PLoS One 11: e0151894

Matecic M, Smith DL, Pan X, Maqani N, Bekiranov S, Boeke JD \& Smith JS (2010) A microarray-based genetic screen for yeast chronological aging factors. PLoS Genet 6: e1000921

McClure JM, Wierman MB, Maqani N \& Smith JS (2012) Isonicotinamide enhances Sir2 protein-mediated silencing and longevity in yeast by raising intracellular NAD ${ }^{+}$ concentration. J Biol Chem 287: 20957-20966

Mitsui A \& Suzuki S (1969) Immunosuppressive effect of Mycophenolic acid. J Antibiot (Tokyo) 22: $358-363$

Mizuguchi G, Shen X, Landry J, Wu WH, Sen S \& Wu C (2004) ATP-driven exchange of histone H2AZ variant catalyzed by SWR1 chromatin remodeling complex. Science 303: $343-348$

Mohammad K, Junio JAB, Tafakori T, Orfanos E \& Titorenko VI (2020) Mechanisms that link chronological aging to cellular quiescence in budding yeast. Int J Mol Sci 21: 1-14

Mortimer RK \& Johnston JR (1959) Life span of individual yeast cells. Nature 183: 1751-1752

Moshfegh CM, Collins CW, Gunda V, Vasanthakumar A, Cao JZ, Singh PK, Godley LA \& Case AJ (2019) Mitochondrial superoxide disrupts the metabolic and epigenetic landscape of $\mathrm{CD}^{+}$and $\mathrm{CD}^{+}$T-lymphocytes. Redox Biol 27:101141

Niccoli T \& Partridge L (2012) Ageing as a risk factor for disease. Curr Biol 22: R741-R752 
Orlandi I, Pellegrino Coppola D, Strippoli M, Ronzulli R \& Vai M (2017) Nicotinamide supplementation phenocopies SIR2 inactivation by modulating carbon metabolism and respiration during yeast chronological aging. Mech Ageing Dev 161: 277-287

Osório H, Silles E, Maia R, Peleteiro B, Moradas-Ferreira P, Günther Sillero MA \& Sillero A (2005) Influence of chronological aging on the survival and nucleotide content of Saccharomyces cerevisiae cells grown in different conditions: occurrence of a high concentration of UDP-N-acetylglucosamine in stationary cells grown in $2 \%$ glucose. FEMS Yeast Res 5: 387-398

Park J-I, Grant CM \& Dawes IW (2005) The high-affinity cAMP phosphodiesterase of Saccharomyces cerevisiae is the major determinant of cAMP levels in stationary phase: involvement of different branches of the Ras-cyclic AMP pathway in stress responses. Biochem Biophys Res Commun 327: 311-319

Parsons AB, Brost RL, Ding H, Li Z, Zhang C, Sheikh B, Brown GW, Kane PM, Hughes TR \& Boone C (2004) Integration of chemical-genetic and genetic interaction data links bioactive compounds to cellular target pathways. Nat Biotechnol 22: 62-69

Postnikoff SDL \& Harkness TAA (2014) Replicative and chronological life-span assays. Methods Mol Biol 1163: 223-227

Powers RW, Kaeberlein M, Caldwell SD, Kennedy BK \& Fields S (2006) Extension of chronological life span in yeast by decreased TOR pathway signaling. Genes Dev 20: 174184

Qiu Y, Fairbanks LD, Rückemann K, Hawrylowicz CM, Richards DF, Kirschbaum B \& Simmonds HA (2000) Mycophenolic acid-induced GTP depletion also affects ATP and 
pyrimidine synthesis in mitogen-stimulated primary human T-lymphocytes. Transplantation 69: $890-897$

Sánchez-Férez F, Ejarque D, Calvet T, Font-Bardia M \& Pons J (2019) Isonicotinamide-based compounds: From cocrystal to polymer. Molecules 24: 4169

Santisteban MS, Hang M \& Smith MM (2011) Histone variant H2A.Z and RNA polymerase II transcription elongation. Mol Cell Biol 31: 1848-1860

Santisteban MS, Kalashnikova T \& Smith MM (2000) Histone H2A.Z regulates transcription and is partially redundant with nucleosome remodeling complexes. Cell 103: 411-422

Santos SM, Laflin S, Broadway A, Burnet C, Hartheimer J, Rodgers J, Smith DL \& Hartman IV JL (2020) High-resolution yeast quiescence profiling in human-like media reveals complex influences of auxotrophy and nutrient availability. GeroScience 43: 941-964

Sarnoski EA, Liu P \& Acar M (2017) A high-throughput screen for yeast replicative lifespan identifies lifespan-extending compounds. Cell Rep 21: 2639-2646

Sauve AA, Moir RD, Schramm VL \& Willis IM (2005) Chemical activation of Sir2-dependent silencing by relief of nicotinamide inhibition. Mol Cell 17: 595-601

Sauve AA \& Schramm VL (2003) Sir2 regulation by nicotinamide results from switching between base exchange and deacetylation chemistry. Biochemistry 42: 9249-9256

Shukla SK, Gunda V, Abrego J, Haridas D, Mishra A, Souchek J, Chaika N V., Yu F, Sasson AR, Lazenby AJ, et al (2015) MUC16-mediated activation of mTOR and c-MYC reprograms pancreatic cancer metabolism. Oncotarget 6: 19118-19131

Sims JL, Sikorski GW, Catino DM, Berger SJ \& Berger NA (1982) 
Poly(adenosinediphosphoribose) Polymerase inhibitors stimulate unscheduled deoxyribonucleic acid synthesis in normal human lymphocytes. Biochemistry 21: 18131821

Smith Jr DL, Maharrey CH, Carey CR, White RA \& Hartman IV JL (2016) Gene-nutrient interaction markedly influences yeast chronological lifespan. Exp Gerontol 86: 113-123

Smith Jr DL, McClure JM, Matecic M \& Smith JS (2007) Calorie restriction extends the chronological lifespan of Saccharomyces cerevisiae independently of the Sirtuins. Aging Cell 6: 649-662

Smith ED, Tsuchiya M, Fox LA, Dang N, Hu D, Kerr EO, Johnston ED, Tchao BN, Pak DN, Welton KL, et al (2008) Quantitative evidence for conserved longevity pathways between divergent eukaryotic species. Genome Res 18: 564-570

Stephan J, Franke J \& Ehrenhofer-Murray AE (2013) Chemical genetic screen in fission yeast reveals roles for vacuolar acidification, mitochondrial fission, and cellular GMP levels in lifespan extension. Aging Cell 12: 574-583

Sweeney MJ, Hoffman DH \& Esterman MA (1972) Metabolism and biochemistry of mycophenolic acid. Cancer Res 32: 1803-1809

Toth B (1983) Lack of carcinogenicity of nicotinamide and isonicotinamide following lifelong administration to mice. Oncology 40: 72-75

Ueda K, Banasik M, Nakajima S, Yook HY \& Kido T (1995) Cell differentiation induced by poly(ADP-ribose) synthetase inhibitors. Biochimie 77: 368-373

Váchová L, Cáp M \& Palková Z (2012) Yeast colonies: a model for studies of aging, 
environmental adaptation, and longevity. Oxid Med Cell Longev 2012: 601836

Wagih O, Usaj M, Baryshnikova A, VanderSluis B, Kuzmin E, Costanzo M, Myers CL,

Andrews BJ, Boone CM \& Parts L (2013) SGAtools: one-stop analysis and visualization of array-based genetic interaction screens. Nucleic Acids Res 41: W591-W596

Wei M, Fabrizio P, Hu J, Ge H, Cheng C, Li L \& Longo VD (2008) Life span extension by calorie restriction depends on Rim15 and transcription factors downstream of Ras/PKA, Tor, and Sch9. PLoS Genet 4: e13

Wierman MB, Maqani N, Strickler E, Li M \& Smith JS (2017) Caloric restriction extends yeast chronological life span by optimizing the Snf1 (AMPK) Signaling Pathway. Mol Cell Biol 37: e00562-16

Wierman MB, Matecic M, Valsakumar V, Li M, Smith Jr DL, Bekiranov S \& Smith JS (2015a) Functional genomic analysis reveals overlapping and distinct features of chronologically long-lived yeast populations. Aging (Albany NY) 7: 177-194

Wierman MB \& Smith JS (2014) Yeast sirtuins and the regulation of aging. FEMS Yeast Res 14: $73-88$

Winzeler EA, Shoemaker DD, Astromoff A, Liang H, Anderson K, Andre B, Bangham R, Benito R, Boeke JD, Bussey H, et al (1999) Functional characterization of the $S$. cerevisiae genome by gene deletion and parallel analysis. Science 285: 901-906

Wogulis M, Chew ER, Donohoue PD \& Wilson DK (2008) Identification of formyl kynurenine formamidase and kynurenine aminotransferase from Saccharomyces cerevisiae using crystallographic, bioinformatic and biochemical evidence. Biochemistry 47: 1608-1621 
Wu WH, Wu CH, Ladurner A, Mizuguchi G, Wei D, Xiao H, Luk E, Ranjant A \& Wu C (2009) $\mathrm{N}$ terminus of Swr1 binds to histone $\mathrm{H} 2 \mathrm{AZ}$ and provides a platform for subunit assembly in the chromatin remodeling complex. J Biol Chem 284: 6200-6207

Xie MW, Jin F, Hwang H, Hwang S, Anand V, Duncan MC \& Huang J (2005) Insights into TOR function and rapamycin response: Chemical genomic profiling by using a high-density cell array method. Proc Natl Acad Sci U S A 102: 7215-7220

Yadav B, Wennerberg K, Aittokallio T \& Tang J (2015) Searching for drug synergy in complex dose-response landscapes using an interaction potency model. Comput Struct Biotechnol J 13: 504-513

Yin N, Ma W, Pei J, Ouyang Q, Tang C \& Lai L (2014) Synergistic and antagonistic drug combinations depend on network topology. PLoS One 9: e93960

\section{FIGURE LEGENDS}

Figure 1. INAM extends chronological lifespan. A) Chemical structures of nicotinamide and isonicotinamide. B) CLS assays with increasing dosage of INAM added to BY4741 at the time of inoculation. C) CLS assay comparing the effect of $25 \mathrm{mM}$ INAM on the auxotrophic strain BY4741 and prototrophic strain FY4, added at inoculation. D) CLS of BY4741 when $25 \mathrm{mM}$ INAM is added $96 \mathrm{hr}$ after inoculation. E) WT (BY4741) or sir2 $\Delta$ hst $1 \Delta$ double mutant CLS with or without $25 \mathrm{mM}$ INAM supplemented at time of inoculation. Viability for each CLS assay is calculated as the percentage of colony forming units at each time point compared to the stationary phase day 3 time point, which was collected 72 hours after inoculation. Error bars indicate standard deviations ( $\mathrm{n}=3$ biological replicates). Statistics are provided in Table S3. 
Figure 2. Chemical genetic screen for INAM sensitive gene deletion mutants in the yeast knockout (YKO) strain collection. A) Spot test growth assay of BY4741 on SC agar plates containing $0,25,50,75$, or 125 mM INAM. B) The haploid yeast knockout collection was pinned onto control SC plates or SC plates with increasing concentrations of INAM using a manual pinning tool. C) Zoomed in portion of plate 7 showing INAM sensitivity of the tho2A strain (yellow arrow). D) Scatter plot of scores from each deletion strain obtained in experiment 1 (x-axis) and experiment 2 (y-axis). Strains with scores lower than -0.3 in both replicates were considered sensitive to a given INAM concentration (representative graph for 75 mM INAM). E) Venn diagram showing the overlap of strains scored as sensitive between different INAM concentrations.

Figure 3. Strains defective for the H2A.Z deposition by SWR1 are sensitive to INAM. A) SWR1 complex schematic diagram illustrating subunits and their sensitivity to INAM. B) Spot test growth assay showing confirmation of INAM sensitivity of $h t z 1 \Delta$ and $s w r l \Delta$ strains from YKO collection. The WT strain is BY4741. C) CLS assay of BY4741 and swr 1 $1 \Delta$ mutant strains supplemented with $25 \mathrm{mM}$ INAM. D) CLS assay of BY4741 and $h t z 1 \Delta$ strains supplemented with 25 mM INAM. Controls were supplemented with equal volume of sterile water instead of INAM stock solution.

Figure 4. Serine biosynthesis mutants are sensitive to INAM. A) Schematic of serine, aspartate, threonine, and cysteine synthesis pathways and their conversion at the one carbon metabolism pathway and purine metabolism. Deleted genes conferring INAM sensitivity are in bold. B) CLS assay showing moderate lifespan extension of $\operatorname{ser} 2 \Delta$ mutant, and full extension by $25 \mathrm{mM}$ INAM. C) Spot test growth assay for WT (BY4741) and ser2 2 strains on SC plates supplemented with 25, 50, or 75 mM INAM. D) Quantitative dose-response curve illustrating the 
relative viability of BY4741 (WT) and the ser $2 \Delta$ mutant at a range of INAM concentrations.

Dots represent values obtained from three biological replicates with sigmoid curves fitted to the data using a dose response function and the Levenberg Marquardt iteration algorithm. Shaded areas around the curve are $95 \%$ confidence bands. E) Bar graph depicting $\mathrm{IC}_{50}$ of BY4741 (WT) and the ser $2 \Delta$ mutant calculated from the equations of fitted curves in panel D. Error bars represent $95 \%$ confidence intervals.

Figure 5. Mycophenolic acid and INAM operate through related but distinct mechanisms. A) Venn diagram depicting a partial overlap between INAM- and MPA-sensitive deletion strains identified in a chemogenomic screen and confirmed by spot assays. B) Synergy map depicting interaction between INAM and MPA in reducing cell growth. Graph is representative of three independent experiments. C) CLS assay with BY4741 showing CLS extension by MPA supplementation (1 or $10 \mu \mathrm{g} / \mathrm{ml}$ ), and suppression of the effect by $0.1 \mathrm{mM}$ guanine. D) CLS assay with BY4741 showing INAM extension of CLS is that is not suppressed by guanine.

Figure 6. Alterations of intracellular nucleotide metabolite levels by 25 mM INAM. A) Summary of quantitative mass spectrometry analysis for a panel of purine and pyrimidine nucleotides and precursors. Shaded blocks (green-up, red-down) indicate metabolites with significant differences $(\mathrm{p}<0.05)$ in INAM treated cells compared to control. Darker shading indicates more significant p-values. B) Examples of significantly reduced purine nucleosides or nucleotides reduced by INAM at each time point. $* \mathrm{p}<0.05, * * \mathrm{p}<0.01$. C) CLS assay showing partial lifespan extension when BY4741 (uracil auxotroph) is supplemented with 4x $(0.8 \mathrm{mM})$ the normal concentration of uracil $(0.2 \mathrm{mM})$ in SC medium. INAM (25 mM) fully extends CLS in combination with $4 \mathrm{x}$ uracil. 


\section{SUPPLEMENTAL FIGURE LEGENDS}

Figure S1. Confirmation of candidate mutants from the INAM sensitivity screen using spot test growth assays. The normalized growth scores from SGAtools are indicated for the two independent screens (performed sequentially). Scores for 25, 50, and $75 \mathrm{mM}$ plates were from day 2 images, while scores for $125 \mathrm{mM}$ plates were from day 3 images. Scores below the -0.3 cutoff are shaded in green.

Figure S2. Confirmation of additional candidate mutants from INAM sensitivity screen. A) Mutants that confirmed as INAM sensitive. Each experiment has its own BY4741 WT control. B) Mutants that did not confirm as INAM sensitive. SGAtools scores for the two screens are indicated to the right, as in Figure S1.

Figure S3. Strains not identified by the screen but confirmed as sensitive in the spot test growth assays. These additional strains were chosen based on identity of confirmed strains from the screen and the literature.

Figure S4. Loss of $B N A 7$ confers INAM resistance and extends CLS. A) Spot test growth assay of candidate INAM-resistant mutants chosen from the screen on $125 \mathrm{mM}$ INAM plates. The only mutant confirmed as resistant was bna7D. B) Schematic diagram of de novo $\mathrm{NAD}^{+}$synthesis and salvage pathways in S. cerevisiae. The Bna7 reaction releases formate from L-formylkynurenine as products. C) CLS assay showing late stage lifespan extension of the bna7D mutant, and further extension by $25 \mathrm{mM}$ INAM.

Figure S5. INAM sensitivity of serine and threonine synthesis mutants (ser $2 \Delta$, thr $1 \Delta$ and hom 34 ) is rescued by the addition of respective amino acids. Error bars represent $95 \%$ confidence intervals derived from the dose-response curves. 
Figure S6. Summary of purine nucleotide and metabolite levels and related gene expression changes induced by INAM in cells collected at $\log$ phase, after $24 \mathrm{hr}$, or $96 \mathrm{hr}$ of liquid culture. Diagram was produced using VANTED.

Figure S7. Pyrimidine metabolite levels and gene expression changes at day 8. A) Summary of pyrimidine nucleotide and metabolite levels and related gene expression from log phase to $96 \mathrm{hr}$ cultures. B) INAM-induced expression changes of select genes involved in nucleotide metabolism. Expression of each gene at day 8 was normalized to TAF10. Three biological replicates were tested. 
A

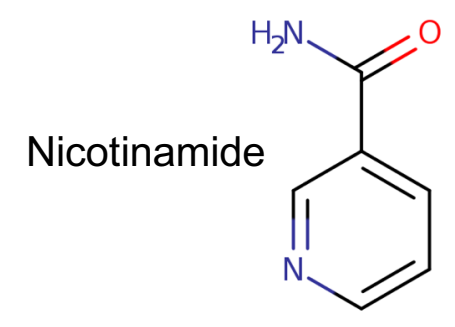

B

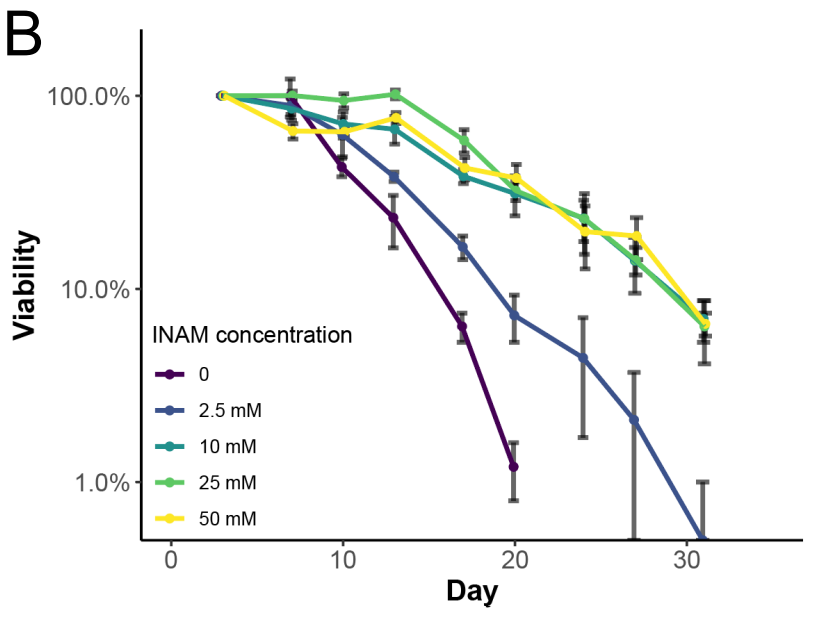

D

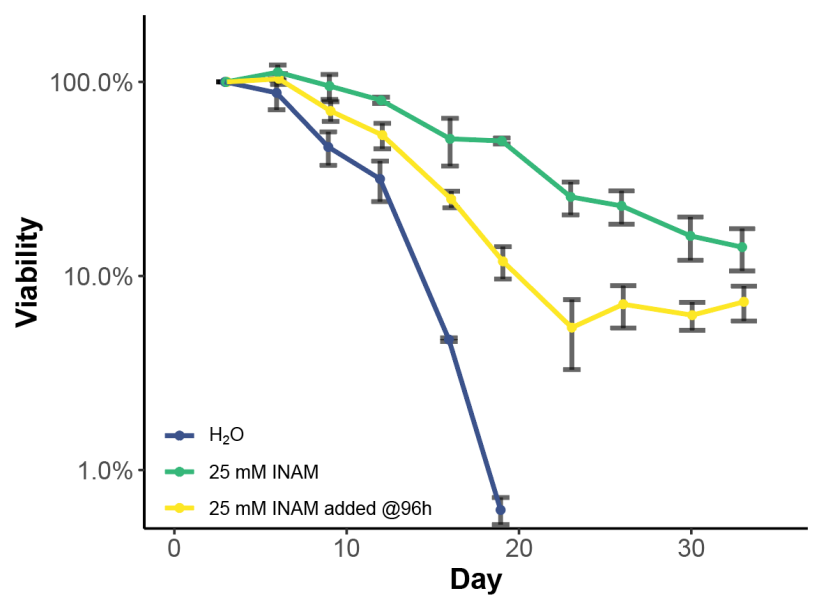<smiles>NC(=O)c1ccncc1</smiles>

C

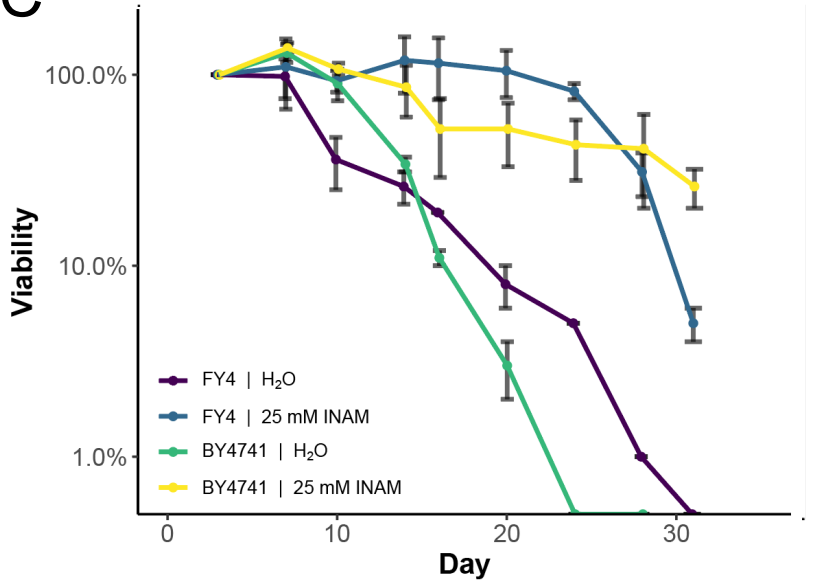

$E$

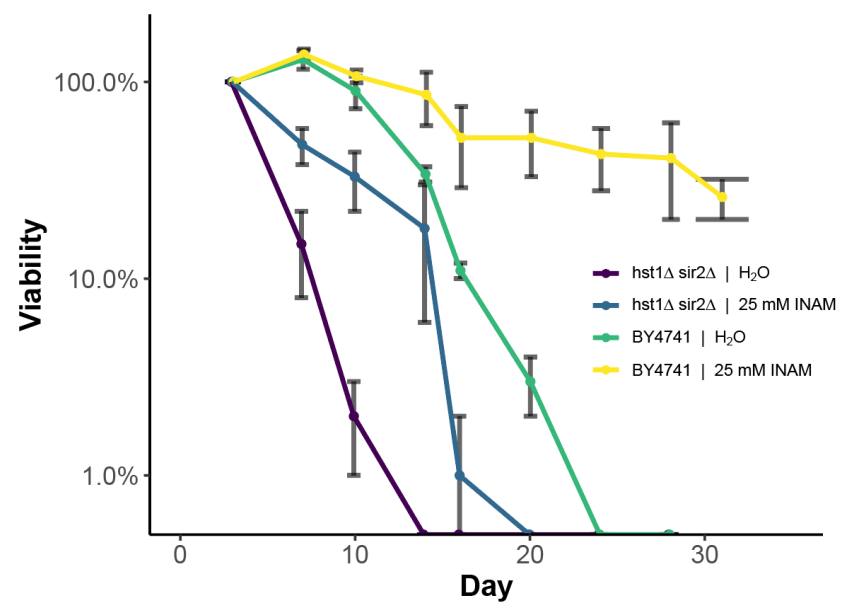


A

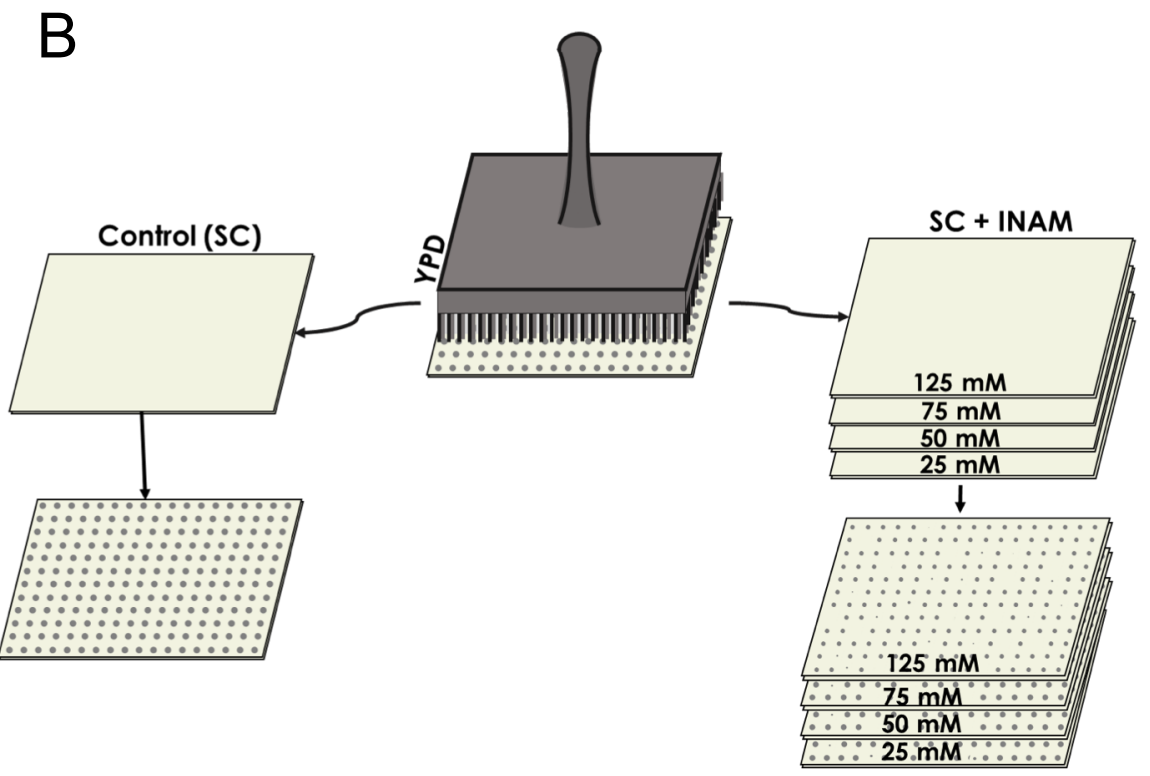

C

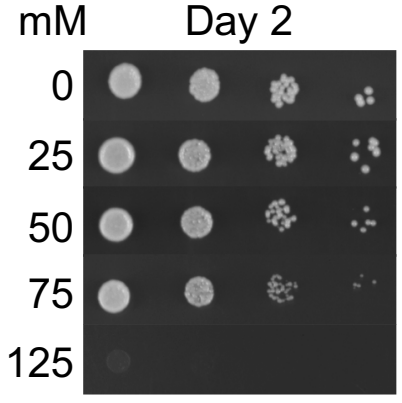

Day 5

125

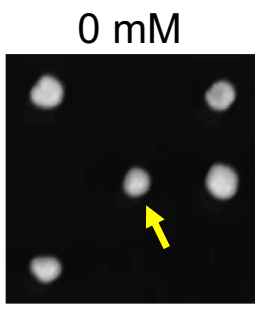

D

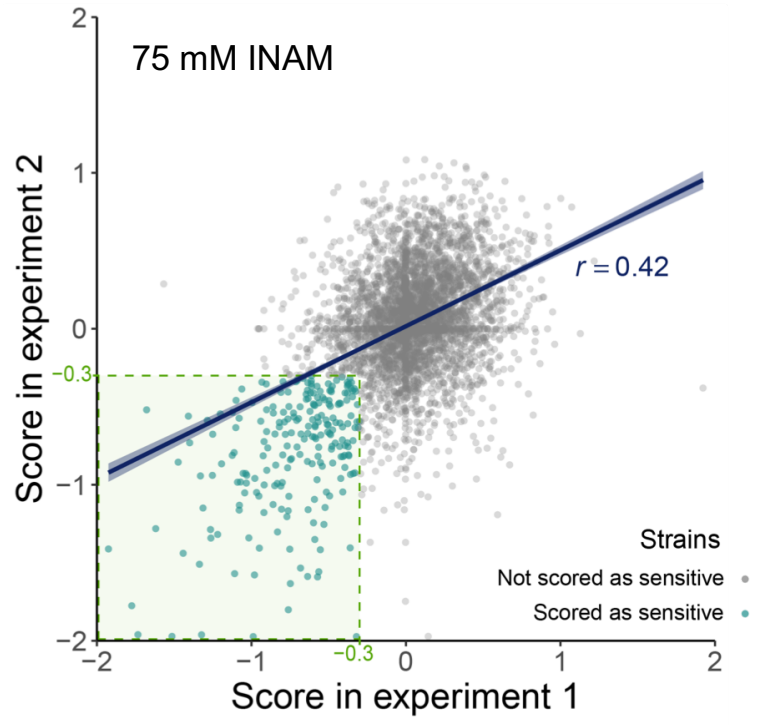

$E$

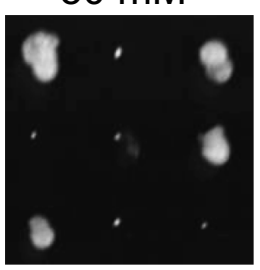

$125 \mathrm{mM}$

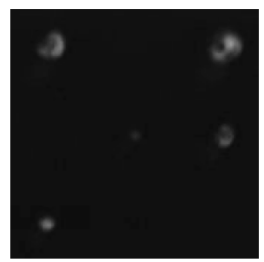

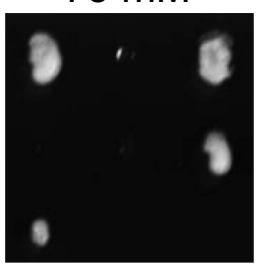

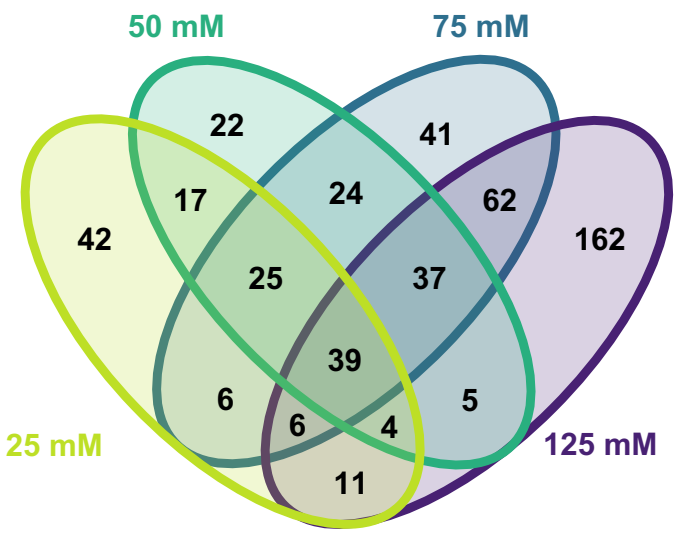




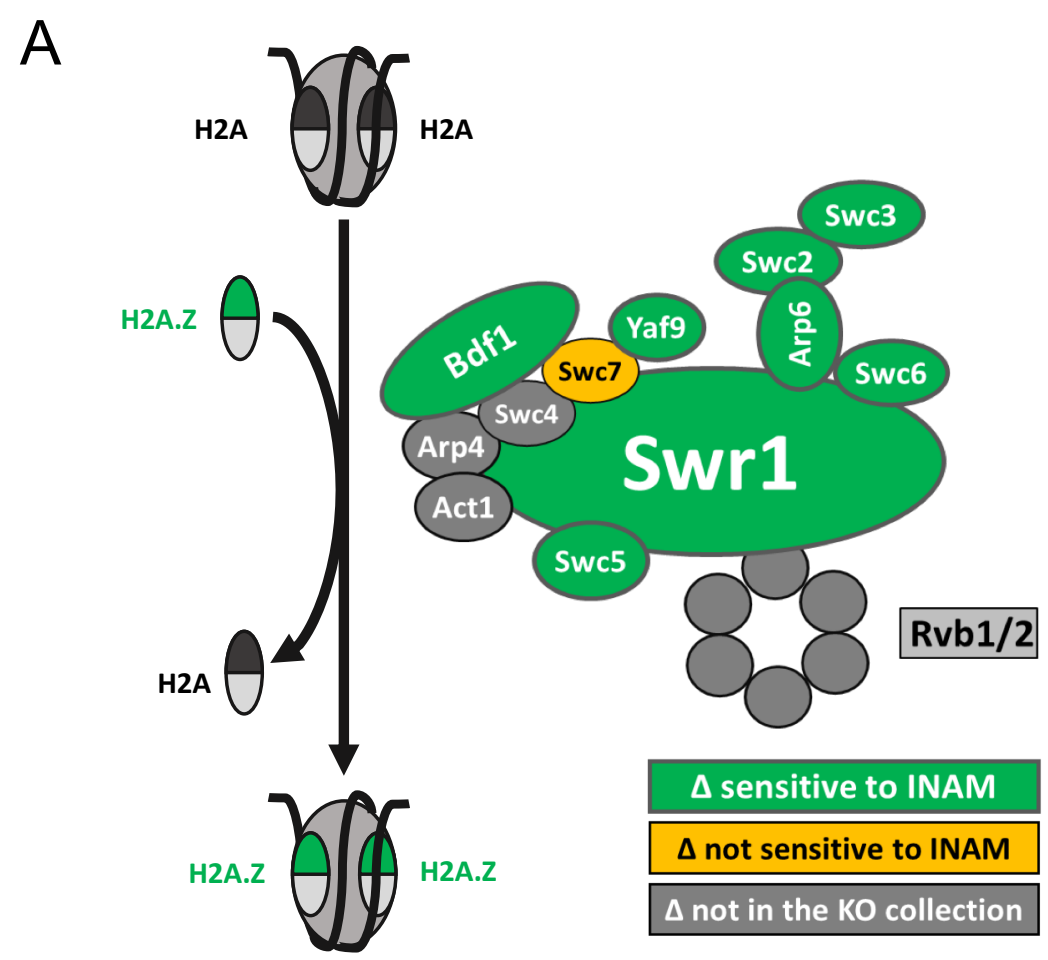

B

\begin{tabular}{|c|c|c|c|c|c|c|}
\hline ORFA & Genes & sc & & $\mathrm{SC}+25 \mathrm{mM}$ INAM & $\mathrm{SC}+50 \mathrm{mM}$ INAM & $\mathrm{SC}+75 \mathrm{mM}$ INAM \\
\hline \multicolumn{2}{|c|}{ BY4741 } & 000 & 0 का & 000 然 & 000 的 & 000 \\
\hline YoL012C & HTZ1 & 000 & 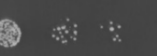 & 000 & & \\
\hline YDR334W & SWR1 & 0006 & $\%$ & $000 \%$ & 000 & () \\
\hline
\end{tabular}
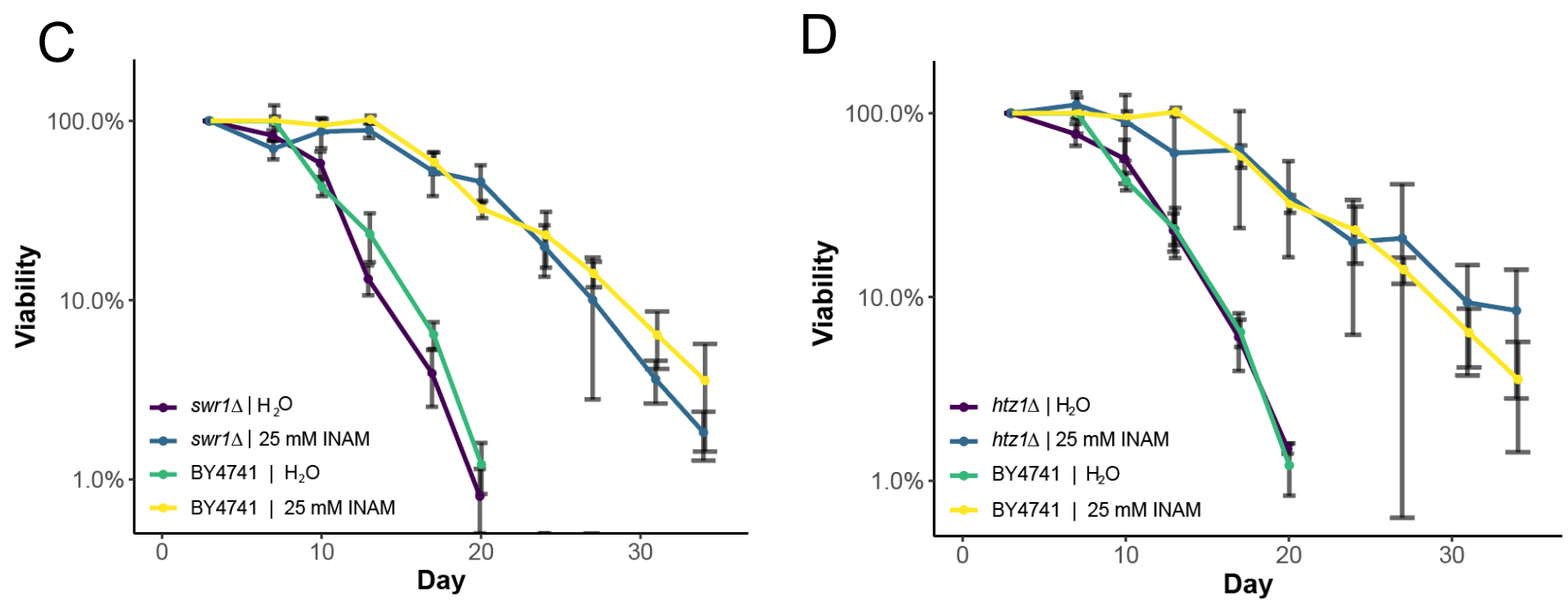
A

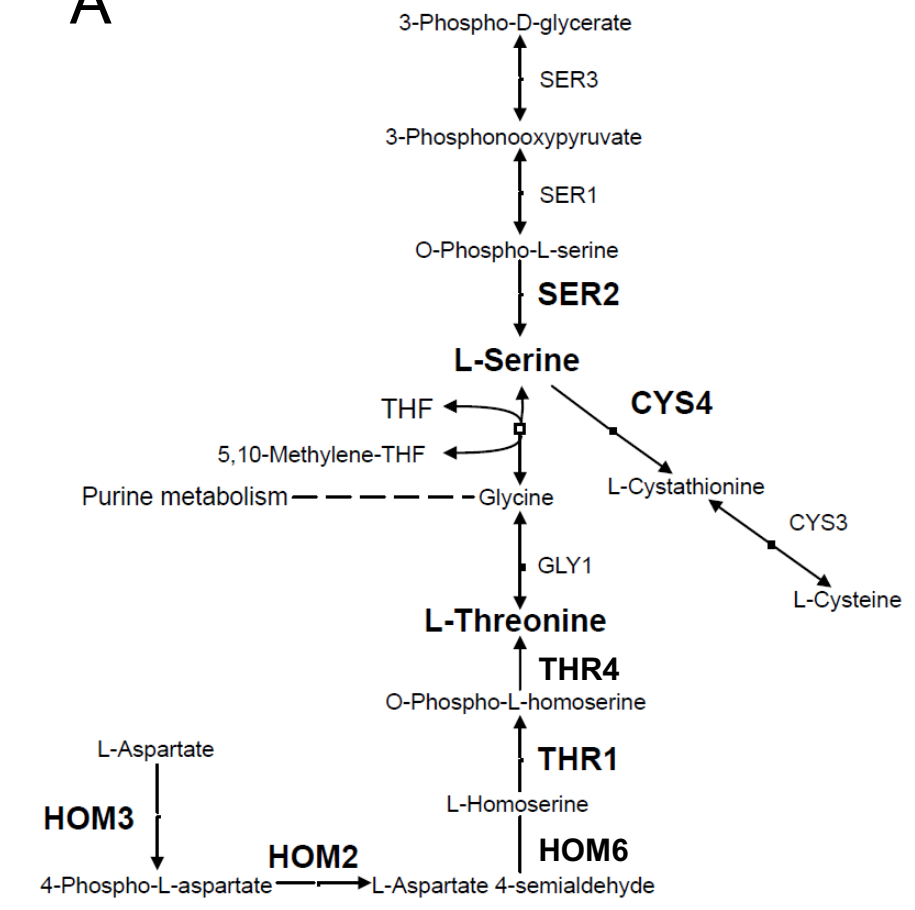

B

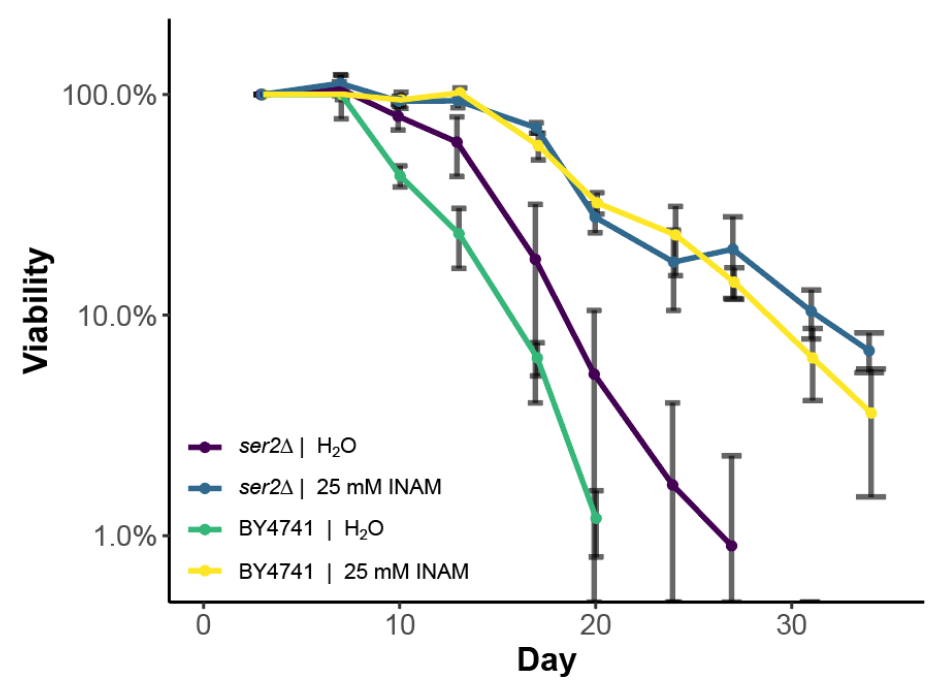

C

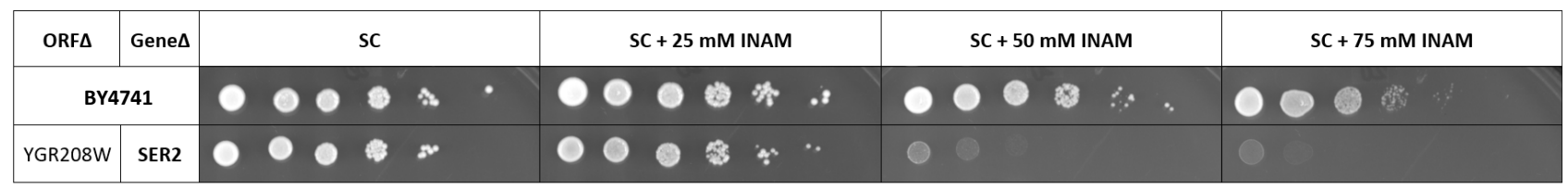

$D$

E
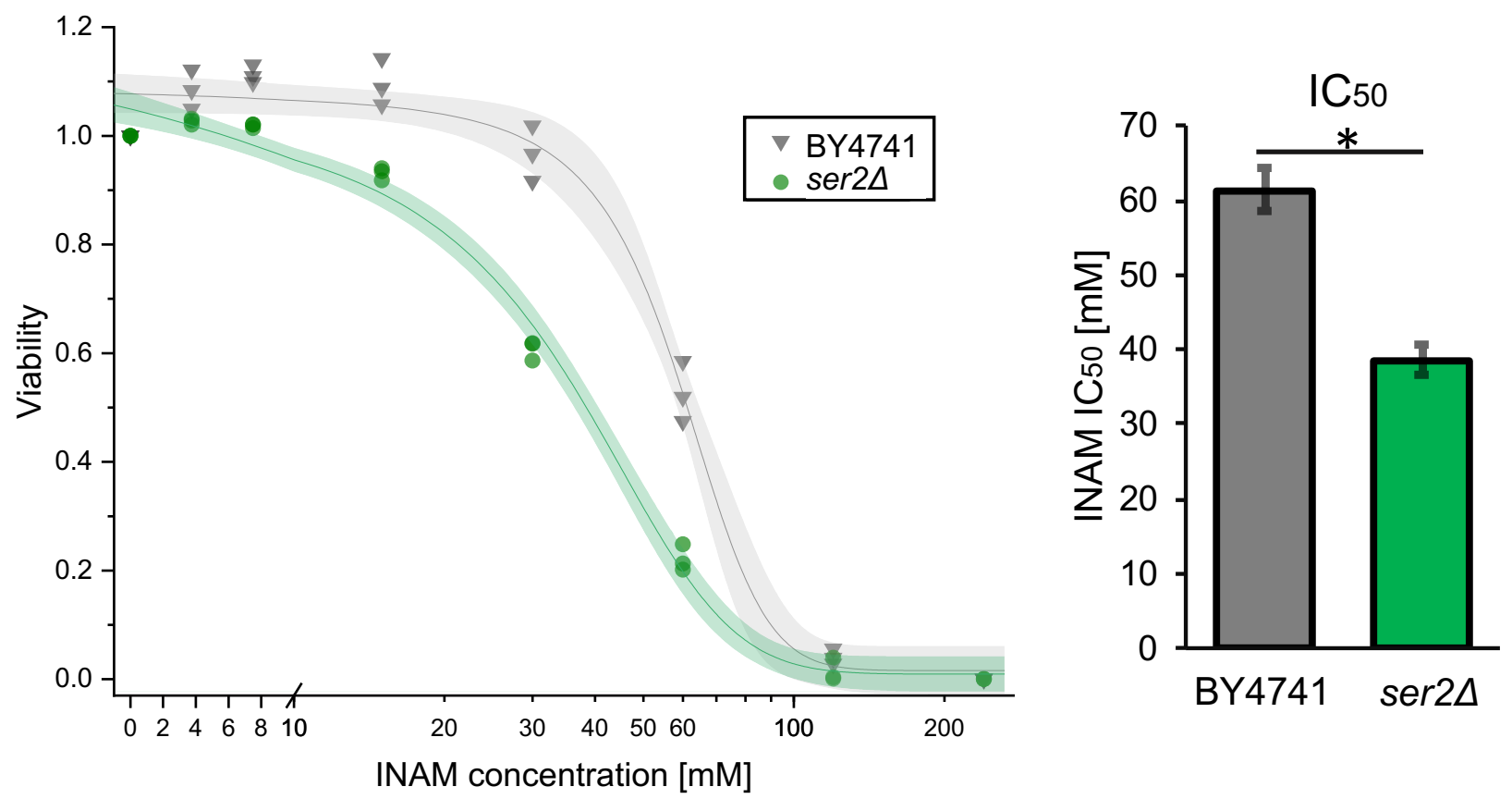
A

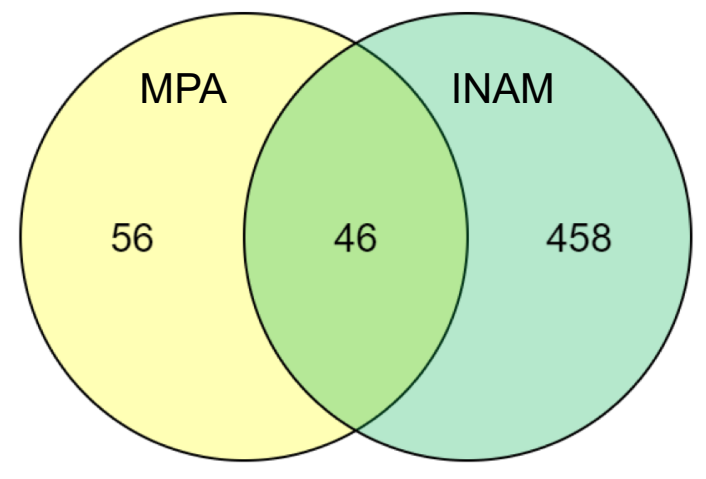

Most synergystic area

Score: 9.86 (ZIP)

C

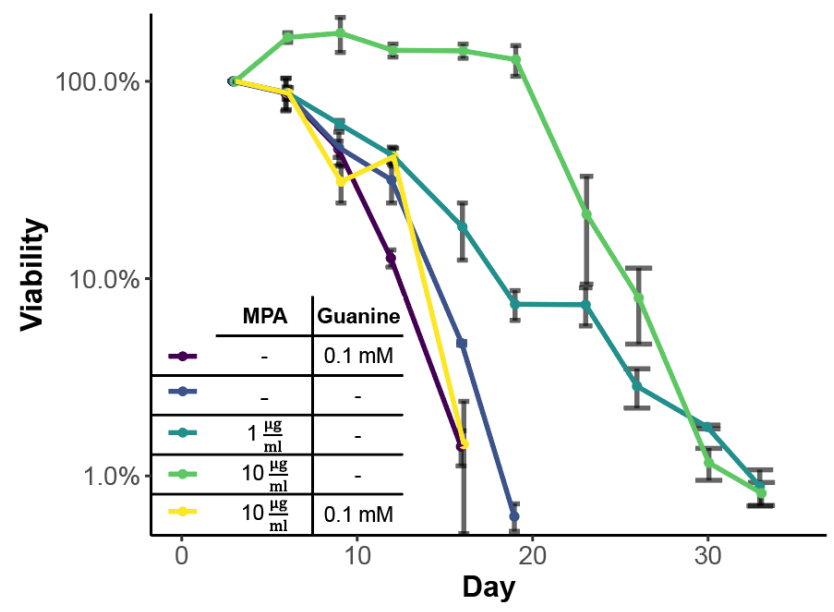

B

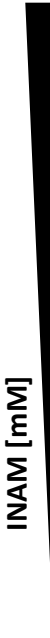
ZIP synergy score: 1.359

120

$\begin{array}{lllll}-20 & -10 & 0 & 10 & 20\end{array}$

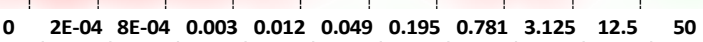

MPA $[\mu \mathrm{M}]$

D

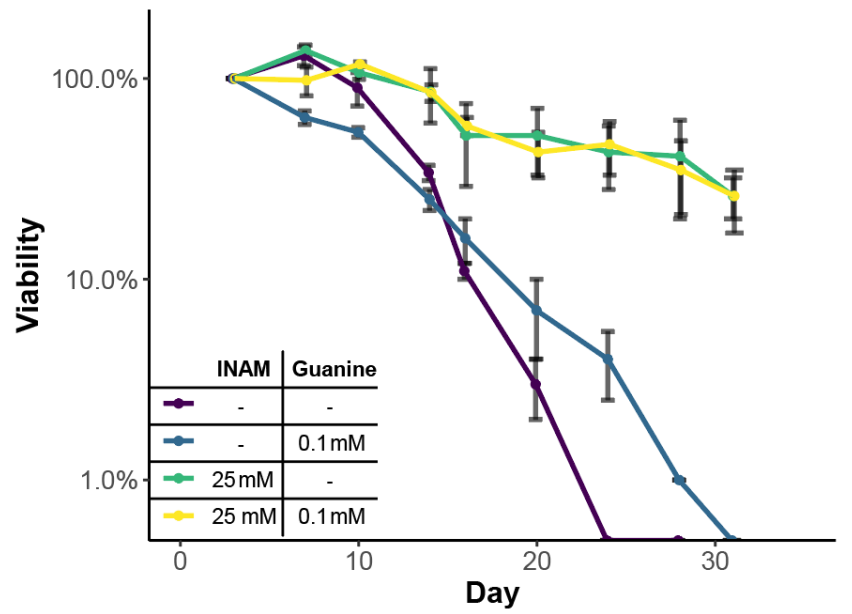


Figure 6

A

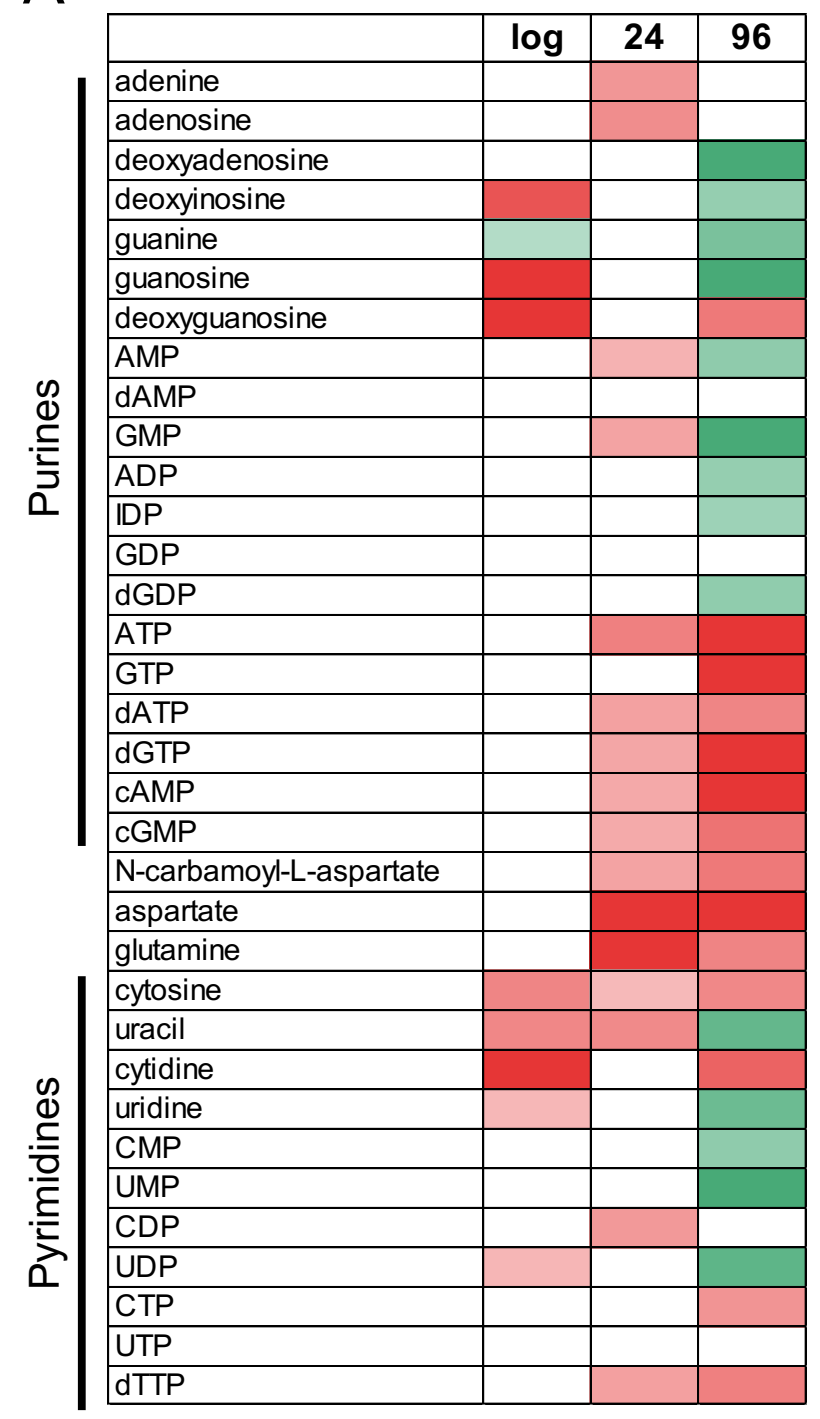
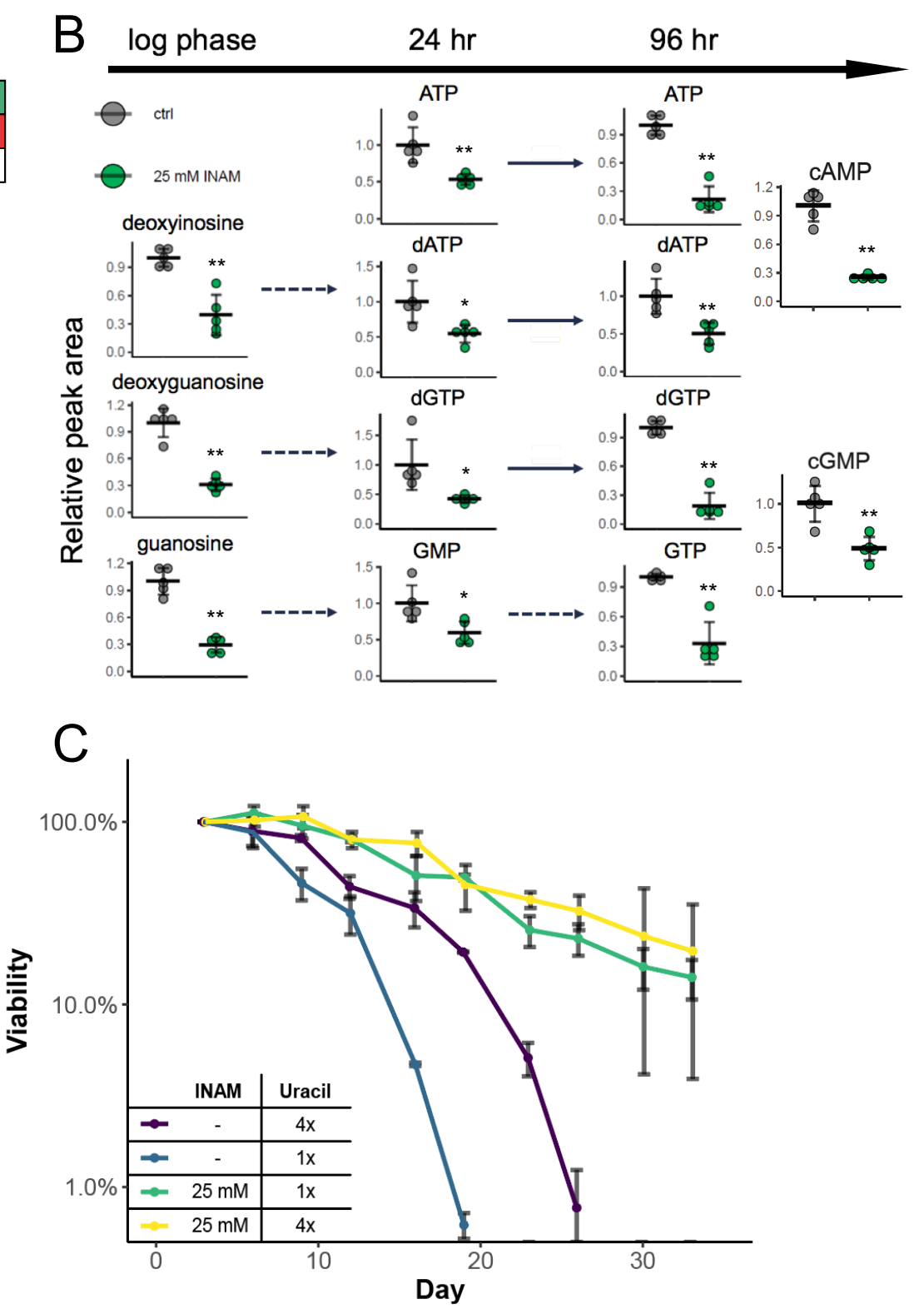